\title{
EFFECTIVE EQUIDISTRIBUTION OF $S$-INTEGRAL POINTS ON SYMMETRIC VARIETIES
}

\author{
YVES BENOIST AND HEE OH
}

\begin{abstract}
Let $K$ be a global field of characteristic not 2. Let $\mathbf{Z}=\mathbf{H} \backslash \mathbf{G}$ be a symmetric variety defined over $K$ and $S$ a finite set of places of $K$. We obtain counting and equidistribution results for the $S$-integral points of $\mathbf{Z}$.

Our results are effective when $K$ is a number field.
\end{abstract}

\section{INTRODUCTION}

1.1. General overview. Consider a finite system of polynomial equations with integral coefficients. Its set of solutions defines an arithmetic variety $\mathbf{Z} \subset \mathbb{C}^{d}$ defined over $\mathbb{Z}$. For a set $S$ of primes including the infinite prime $\infty$, let $\mathbb{Z}_{S}$ denote the ring of $S$-integers of $\mathbb{Q}$, that is, the set of rational numbers whose denominators are products of primes in $S$. If $S=\{\infty\}, \mathbb{Z}_{S}$ is simply the ring of integers $\mathbb{Z}$, and if $S$ consists of all the primes, then $\mathbb{Z}_{S}$ is the field $\mathbb{Q}$ of rational numbers. For any subring $R$ of $\mathbb{C}$, we denote by $\mathbf{Z}_{R}$ the set of points in $\mathbf{Z}$ with coordinates in $R$. One of the fundamental questions in number theory is to understand the properties of sets $\mathbf{Z}_{\mathbb{Z}_{S}}$. In this paper, we obtain effective counting and equidistribution results of the $S$-integral points, for $S$-finite, in the case when $\mathbf{Z}$ is a symmetric variety.

The counting question in this set-up has been completely solved for the integral points via several different methods. The first solution is due to Duke, Rudnick and Sarnak in 1993 [15] and their proof uses the theory of automorphic forms. Almost at the same time, Eskin and McMullen gave the second proof utilizing mixing properties of semisimple real algebraic groups [17]. The third proof, due to Eskin, Mozes and Shah [18, is based on the ergodic theory of flows on homogeneous spaces, more precisely, Ratner's work on the unipotent flows.

The approach of [17] using mixing properties has several advantages over the others in our viewpoint. First it does not require the deep theory of automorphic forms, avoiding technical difficulties in dealing with the Eisenstein series as in [15]. Secondly, although this was never addressed in [17], in principle it also gives a rate of convergence which the ergodic method of [18] does not give. Thirdly the method can be extended to other global fields of positive characteristic, which is again hard to be achieved via the ergodic method.

the second author is partially supported by NSF grant 0629322 . 
For these reasons, we develop the approach of Eskin and McMullen [17] in this paper in order to obtain effective results for the general $S$-integral points on symmetric varieties.

We use the mixing properties of $S$-algebraic semisimple groups, with a rate of convergence. Implementing this in the counting problem, a crucial technical ingredient is to verify certain geometric property, which was named the wavefront property by [17], for an $S$-algebraic symmetric variety. We prove this using the polar decompositions for non-archimedean symmetric spaces obtained in [4] specifically for this purpose. We emphasize that the wave front property is precisely the reason that our proofs work in the setting of an $S$-algebraic symmetric variety for $S$ finite. This property does not hold for a general homogeneous variety even over the reals. In obtaining effective counting results for the $S$-integral points of bounded height, we also use the works of Denef on $p$-adic local zeta functions ([12, [13]) and of Jeanquartier on fiber integrations [30].

We remark that the approach for counting via mixing was initiated in 1970 by Margulis in his dissertation on Anosov dynamical systems [34. Recently similar mixing properties in an adelic setting have been used in the study of rational points of group varieties (see [8], [22], and [27]). We also mention that for the case of group varieties, the effective counting result was obtained for integral points in [23] and [37. We refer to [31], [26], [20], [16], [25], [19], [36] etc., for other types of counting and equidistribution results.

1.2. Main results. We now give a precise description of the main results of this paper.

Let $K$ be a global field of characteristic not 2, i.e. a finite extension of $\mathbb{Q}$ or of $\mathbb{F}_{q}(t)$ where $q$ is an odd prime. Let $\mathbf{Z}$ be a symmetric variety in a vector space $\mathbf{V}$ defined over $K$. That is, there exist a connected algebraic almost $K$-simple group G defined over $K$, a $K$-representation $\rho: \mathbf{G} \rightarrow \mathbf{G L}(\mathbf{V})$ with finite kernel and a non-zero point $z_{0} \in \mathbf{V}_{K}$ whose stabilizer $\mathbf{H}$ in $\mathbf{G}$ is a symmetric $K$-subgroup of $\mathbf{G}$ such that $\mathbf{Z}=z_{0} \mathbf{G}$. By a symmetric $K$-subgroup of $\mathbf{G}$, we mean a $K$-subgroup whose identity component coincides with the identity component of the group of fixed points $\mathbf{G}^{\sigma}$ for an involution $\sigma$ of $\mathbf{G}$ defined over $K$. We assume that the identity component $\mathbf{H}^{0}$ has no non-trivial $K$-character.

We fix a basis of the $K$-vector space $\mathbf{V}_{K}$ so that one can define, for any subring $\mathcal{O}$ of $K$, the subsets $\mathbf{V}_{\mathcal{O}} \subset \mathbf{V}, \mathbf{Z}_{\mathcal{O}} \subset \mathbf{Z}$ and $\mathbf{G}_{\mathcal{O}} \subset \mathbf{G}$ of points with coefficients in $\mathcal{O}$. For each place $v$ of $K$, denote by $K_{v}$ be the completion of $K$ with respect to the absolute value $|\cdot|_{v}$. We write $\mathbf{V}_{v}, \mathbf{Z}_{v}$ and $\mathbf{G}_{v}$ for $\mathbf{V}_{K_{v}}, \mathbf{Z}_{K_{v}}$ and $\mathbf{G}_{K_{v}}$, respectively.

Let $S$ be a finite set of places of $K$ containing all archimedean (sometimes called infinite) places with $\mathbf{G}_{v}$ non-compact. Note that if char $\mathrm{K}$ is positive, $K$ does not have any archimedean place. We denote by $\mathcal{O}_{S}$ the ring of $S$-integers of 
$K$, that is,

$$
\mathcal{O}_{S}:=\left\{\left.k \in K|| k\right|_{v} \leq 1 \text { for each finite } v \notin S\right\} .
$$

For instance, if $K=\mathbb{Q}$, we have $\mathcal{O}_{S}=\mathbb{Z}_{S}$. We set $\mathbf{Z}_{S}=\prod_{v \in S} \mathbf{Z}_{v}$ and similarly $\mathbf{G}_{S}$ and $\mathbf{H}_{S}$.

Note that the sets $\mathbf{Z}_{\mathcal{O}_{S}}, \mathbf{G}_{\mathcal{O}_{S}}$ and $\mathbf{H}_{\mathcal{O}_{S}}$ are discrete subsets of $\mathbf{Z}_{S}, \mathbf{G}_{S}$, and $\mathbf{H}_{S}$ respectively, via the diagonal embeddings.

By a theorem of Borel and Harish-Chandra in characteristic 0 and of Behr and Harder in positive characteristic (see Theorem I.3.2.4 in [35]), the subgroups $\mathbf{G}_{\mathcal{O}_{S}}$ and $\mathbf{H}_{\mathcal{O}_{S}}$ are lattices in $\mathbf{G}_{S}$ and $\mathbf{H}_{S}$ respectively. Again, by a theorem of Borel, Harish-Chandra, Behr and Harder, the group $\mathbf{G}_{\mathcal{O}_{S}}$ has only finitely many orbits in $\mathbf{Z}_{\mathcal{O}_{S}}$ (see Theorem 10 in [21]).

Hence our counting and equidistribution question of $S$-integral points of $\mathbf{Z}$ reduces to counting and equidistribution of points in a single $\mathbf{G}_{\mathcal{O}_{S}}$-orbit, say, for instance, in $z_{0} \mathbf{G}_{\mathcal{O}_{S}}$. Set

$$
Z_{S}:=z_{0} \mathbf{G}_{S}=\prod_{v \in S} z_{0} \mathbf{G}_{v}
$$

and let $\Gamma_{S}$ be a subgroup of finite index in $\mathbf{G}_{\mathcal{O}_{S}}$. Let $\mu_{X_{S}}$ be a $\mathbf{G}_{S^{-}}$-invariant measure on $X_{S}:=\Gamma_{S} \backslash \mathbf{G}_{S}$ and $\mu_{Y_{S}}$ an $\mathbf{H}_{S}$-invariant measure on $Y_{S}:=\left(\Gamma_{S} \cap\right.$ $\left.\mathbf{H}_{S}\right) \backslash \mathbf{H}_{S}$. For each $v \in S$, we choose an invariant measure $\mu_{Z_{v}}$ on $Z_{v}:=z_{0} \mathbf{G}_{v}$ so that for $\mu_{Z_{S}}:=\prod_{v \in S} \mu_{Z_{v}}$, we have $\mu_{X_{S}}=\mu_{Z_{S}} \mu_{Y_{S}}$ locally. For a subset $S_{0} \subset S$, we set $\mu_{Z_{S_{0}}}=\prod_{v \in S_{0}} \mu_{Z_{v}}$.

For a Borel subset $B$ of $Z_{S}$, we set

$$
\operatorname{vol}(B):=\frac{\mu_{Y_{S}}\left(Y_{S}\right)}{\mu_{X_{S}}\left(X_{S}\right)} \mu_{Z_{S}}(B) .
$$

We assume that $\mathbf{G}_{S}$ is non-compact; otherwise $\mathbf{Z}_{\mathcal{O}_{S}}$ is finite. By considering a finite covering of $\mathbf{G}$ by its simply connected cover, we may also assume that $\mathbf{G}$ is simply connected without loss of generality.

Before stating our main result, we summarize our set-up:

$K$ is a global field such that $\operatorname{char}(K) \neq 2, \mathbf{Z} \simeq \mathbf{H} \backslash \mathbf{G}$ is a symmetric variety in a vector space $\mathbf{V}$ defined over $K$ where $\mathbf{G}$ is an almost $K$-simple simply-connected $K$-group acting on $\mathbf{V}$ such that the identity component $\mathbf{H}^{0}$ has no non-trivial $K$-character, and $S$ is a finite set of places of $K$ containing all the infinite places $v$ with $\mathbf{G}_{v}$ non-compact and satisfying that $\mathbf{G}_{S}$ is non-compact.

Counting $S$-integral points. We first state our counting results. We refer to Definition 6.1 for the notion of a well-rounded sequence of subsets $B_{n}$ in $Z_{S}$. Roughly speaking, this means that for all small $\varepsilon>0$, the boundaries of $B_{n}$ can be approximated by neighborhoods whose volume is of $\varepsilon$-order compared to the volume of $B_{n}$ uniformly. 
Theorem 1.1. For any well-rounded sequence of subsets $B_{n}$ of $Z_{S}$ with volume tending to infinity, we have

$$
\#\left(z_{0} \Gamma_{S} \cap B_{n}\right) \sim \operatorname{vol}\left(B_{n}\right) \quad \text { as } n \rightarrow \infty .
$$

As a corollary, we obtain that the number of $S$-integral points of size less than $T$ is given by the volume of the corresponding ball in $Z_{S}$. A most natural way to measure the size of an $S$-integral point is given by a height function $\mathrm{H}_{S}$. For $z \in \mathbf{Z}\left(\mathcal{O}_{S}\right)$, it is simply

$$
\mathrm{H}_{S}(z):=\prod_{v \in S}\|z\|_{v}
$$

where the $\|\cdot\|_{v}$ are norms on $\mathbf{V}_{K_{v}}$ which are euclidean when $v$ is infinite and which are max norms when $v$ is finite. This height function $\mathrm{H}_{S}$ naturally extends to $Z_{S}$.

Corollary 1.2. As $T \rightarrow \infty$,

$$
\#\left\{z \in z_{0} \Gamma_{S}: \mathrm{H}_{S}(z)<T\right\} \sim \operatorname{vol}\left(B_{S}(T)\right) .
$$

where $B_{S}(T):=\left\{z \in Z_{S}: \mathrm{H}_{S}(z)<T\right\}$.

When $K$ is a number field, Theorem 1.1 is proved with a rate of convergence (see Theorem 12.2). For instance, we get:

Theorem 1.3. Let $K$ be a number field. There exists $\delta>0$ such that as $T \rightarrow \infty$

$$
\#\left\{z \in z_{0} \Gamma_{S}: \mathrm{H}_{S}(z)<T\right\}=\operatorname{vol}\left(B_{S}(T)\right)\left(1+O\left(T^{-\delta}\right)\right) .
$$

We will see (Remark 7.10) that there exist $a \in \mathbb{Q}_{>0}, b \in \mathbb{Z}_{\geq 0}$ and $c_{1}, c_{2}>0$ such that for $T$ large,

$$
c_{1} T^{a} \log (T)^{b} \leq \operatorname{vol}\left(B_{S}(T)\right) \leq c_{2} T^{a} \log (T)^{b} .
$$

In general, one cannot choose $c_{1}=c_{2}$.

The rate of convergence in Theorem 1.3 is new even for integral points in the generality of symmetric varieties. In this case, as $T \rightarrow \infty, \operatorname{vol}\left(B_{S}(T)\right) \sim$ $c T^{a} \log (T)^{b}$, for some $c>0$.

Our proof of Theorem 1.3 uses Denef's result on local zeta functions which is not available in positive characteristic. This explains our hypothesis on the characteristic of $K$.

Equidistribution of $S$-integral points. To motivate, consider the case when $K=\mathbb{Q}$ and suppose that $\mathbf{Z}_{\mathbb{Z}\left[p^{-1}\right]}$, the set of rational points in $\mathbf{Z}$ with denominators only power of $p$, is a dense subset in $\mathbf{Z}_{\mathbb{R}}$, which is often the case. A natural question is when the sequence of subsets in $\mathbf{Z}_{\mathbb{Z}\left[p^{-1}\right]}$ consisting of elements of denominator precisely $p^{n}$ is equidistributed as $n \rightarrow \infty$. That is, for two compact subsets $\Omega_{1}, \Omega_{2}$ of $\mathbf{Z}_{\mathbb{R}}$, as $n \rightarrow \infty$,

$$
\frac{\left\{x \in \mathbf{Z}_{\mathbb{Q}} \cap \Omega_{1}: p^{n} x \in \mathbf{V}_{\mathbb{Z}}, p \nmid p^{n} x\right\}}{\left\{x \in \mathbf{Z}_{\mathbb{Q}} \cap \Omega_{2}: p^{n} x \in \mathbf{V}_{\mathbb{Z}}, p \nmid p^{n} x\right\}} \sim \frac{\operatorname{vol}\left(\Omega_{1}\right)}{\operatorname{vol}\left(\Omega_{2}\right)} ?
$$


Once we note that $p^{n} x \in V_{\mathbb{Z}}$ is equivalent to the condition that the $p$-adic maximum norm of $x$ is at most $p^{n}$, the above question can be rephrased as the question of equidistribution on $\mathbf{Z}_{\mathbb{R}}$ of the sets $\left\{z \in \mathbf{Z}_{\mathbb{Z}\left[p^{-1}\right]}:\|z\|_{p}=p^{n}\right\}$.

We answer this question in greater generalities:

Theorem 1.4. Let $S=S_{0} \sqcup S_{1}$ be a partition of $S$. For any well-rounded sequence $B_{n}$ of subsets of $Z_{S_{1}}$ with volume tending to infinity, and for any compact subset $\Omega \subset Z_{S_{0}}$ of positive measure and of boundary measure 0 , we have

$$
\# z_{0} \Gamma_{S} \cap\left(\Omega \times B_{n}\right) \sim \frac{\mu_{Y_{S}}\left(Y_{S}\right)}{\mu_{X_{S}}\left(X_{S}\right)} \mu_{Z_{S_{0}}}(\Omega) \mu_{Z_{S_{1}}}\left(B_{n}\right) \quad \text { as } n \rightarrow \infty .
$$

Note that the special case discussed prior to Theorem 1.4 corresponds to $K=$ $\mathbb{Q}, S_{0}=\{\infty\}, S_{1}=\{p\}$, and $B_{n}=\left\{z \in \mathbf{Z}_{\mathbb{Q}_{p}}:\|z\|_{p}=p^{n}\right\}$.

Note that in all the above theorems, we may replace $z_{0} \Gamma_{S}$ by $Z_{\mathcal{O}_{S}}:=Z_{S} \cap \mathbf{Z}_{\mathcal{O}_{S}}$. as long as we renormalize the volume form so that the volume of a subset $E \subset Z_{S}$ is given by

$$
\widetilde{\operatorname{vol}}(E)=\sum \frac{\mu_{Y_{S}}\left(Y_{S}\right)}{\mu_{X_{S}}\left(X_{S}\right)} \mu_{Z_{S}}(E)
$$

where we sum the contributions from each $\Gamma_{S}$-orbit in $Z_{\mathcal{O}_{S}}$. Hence we obtain:

Corollary 1.6. Assume $S$ has at least two places.

(1) For any finite $v \in S$, the sets $Z(T):=\left\{z \in Z_{\mathcal{O}_{S}}:\|z\|_{v}=T\right\}$ become equidistributed in $Z_{S-\{v\}}$ as $T \rightarrow \infty$, subject to the condition $Z(T) \neq \emptyset$.

(2) For an infinite $v \in S$, the sets $Z_{T}:=\left\{z \in Z_{\mathcal{O}_{S}}:\|z\|_{v} \leq T\right\}$ become equidistributed in $Z_{S-\{v\}}$ as $T \rightarrow \infty$, provided $Z_{v}$ is non-compact.

Again, when $K$ is a number field, Theorem 1.4 and Corollary 1.6 are proved with a rate of convergence (see Corollary 12.3 and Proposition 13.2). For instance, we obtain:

Theorem 1.7. Let $K$ be a number field and $S=S_{\infty} \sqcup S_{f}$ be the partition of $S$ into infinite and finite places. We assume that $\mathbf{G}_{S_{f}}$ is non compact. Set $\beta_{T}:=\left\{z \in Z_{S_{f}}: H_{S_{f}}(z)<T\right\}$. Then there exist $\delta>0$ such that for any compact subset $\Omega$ of $Z_{S_{\infty}}$ with piecewise smooth boundary,

$$
\#\left(z_{0} \Gamma_{S} \cap\left(\Omega \times \beta_{T}\right)\right)=\frac{\mu_{Y_{S}}\left(Y_{S}\right)}{\mu_{X_{S}}\left(X_{S}\right)} w_{T} \mu_{Z_{\infty}}(\Omega)\left(1+O\left(T^{-\delta}\right)\right) \quad \text { as } T \rightarrow \infty
$$

where $w_{T}:=\mu_{Z_{S_{f}}}\left(\beta_{T}\right)$.

Equidistribution of translates of $\mathbf{H}_{S}$-orbits. Set $X_{S}=\mathbf{G}_{\mathcal{O}_{S}} \backslash \mathbf{G}_{S}$ and $Y_{S}=$ $\mathbf{H}_{\mathcal{O}_{S}} \backslash \mathbf{H}_{S}$. Let $\mu_{X_{S}}$ and $\mu_{Y_{S}}$ be invariant probability measures on $X_{S}$ and $Y_{S}$ respectively. The following theorem is a crucial tool in proving Theorem 1.1. It states that the translates $Y_{S} g$ is equidistributed in $X_{S}$ as $g$ leaves compact subsets of $\mathbf{H}_{S} \backslash \mathbf{G}_{S}$. 
Theorem 1.8. For any $\psi \in C_{c}\left(X_{S}\right)$,

$$
\int_{Y_{S}} \psi(y g) d \mu_{Y_{S}}(y) \rightarrow \int_{X_{S}} \psi d \mu_{X_{S}} \quad \text { as g tends to infinity in } \mathbf{H}_{S} \backslash \mathbf{G}_{S} \text {. }
$$

The case when $K=\mathbb{Q}$ and $S=\{\infty\}$, Theorem 1.8 was proved in [17], [15], [18] and [45]. In the case when $\mathbf{H}_{S}$ is semisimple and non-compact, it is recently proved in [24], by extending theorems of Mozes-Shah [38] and Dani-Margulis [10] in $S$-algebraic settings. None of the above papers address the rate issues, while our proof gives effective version in the case when $\operatorname{char}(K)=0$ : a smooth function on $X_{S}$ is a function which is smooth for each infinite place in $S$ and invariant under a compact open subgroup of $\mathbf{G}_{v}$ for each finite place $v \in S$. The following effective version of theorem 1.8 is a crucial tool in proving Theorem 1.3 as well as other effective results in this paper.

Theorem 1.9. For $K$ number field, there exists $\kappa>0$ such that, for any smooth function $\psi$ on $X_{S}$ with compact support, there exists $c=c_{\psi}>0$ such that

$$
\left|\int_{Y_{S}} \psi(y g) d \mu_{Y_{S}}(y)-\int_{X_{S}} \psi d \mu_{X_{S}}\right| \leq c \mathrm{H}_{S}\left(z_{0} g\right)^{-\kappa} \quad \text { for all } g \in G_{S} \text {. }
$$

Examples. Let $K=\mathbb{Q}$ and consider the following pairs $(\mathbf{V}, f)$ :

(A) $\mathbf{V}$ : the affine $n$-space with $n \geq 3$ and $f$ : an integral quadratic form of $n$-variables. If $n=3$, we assume that $f$ does not represent 0 over $\mathbb{Q}$.

(B) $\mathbf{V}$ : the space of symmetric $n \times n$ matrices with $n \geq 3$ and $f= \pm \operatorname{det}$.

(C) $\mathbf{V}$ : the space of skew-symmetric $2 n \times 2 n$-matrices with $n \geq 2$ and $f=$ \pm pffaf $= \pm \sqrt{\text { det. }}$

For a positive integer $m$, define

$$
\mathbf{V}_{m}:=\{x \in \mathbf{V}: f(x)=m\} .
$$

Consider the radial projection $\pi: \mathbf{V}_{m} \rightarrow \mathbf{V}_{1}$ given by $x \mapsto m^{1 / d} x$ where $d$ is the degree of $f$. Let $\mathbf{V}(\mathbb{Z})^{\text {prim }}$ be the set of primitive integral vectors in $\mathbf{V}$. For a finite set $S$ of primes of $\mathbb{Q}$ containing the infinite prime $\infty$, we denote by $\langle S\rangle \subset \mathbb{Q}^{*}$ the multiplicative semigroup generated by the finite primes in $S$.

We give a partial answer to the following Linnik problem (see [32], [44, [20], [40], 36]):

Corollary 1.10. Fix $S$ and $(\mathbf{V}, f)$ as above. Then there exist constants $\delta>0$ and $\omega_{m}$, such that for any non-empty compact subset $\Omega \subset \mathbf{V}_{1}(\mathbb{R})$ with piecewise smooth boundary, we have

$$
\# \Omega \cap \pi\left(\mathbf{V}_{m}(\mathbb{Z})^{\text {prim }}\right)=\omega_{m} \operatorname{vol}(\Omega)\left(1+O\left(m^{-\delta}\right)\right)
$$

as $m \rightarrow \infty$ in $\langle S\rangle$, subject to $\mathbf{V}_{m}(\mathbb{Z})^{\text {prim }} \neq \emptyset$. 
A special case of (A) gives an effective equidistribution for $\left\{x \in \mathbb{Z}^{3}: f(x)=m\right\}$ with $f=x^{2}+y^{2}+z^{2}$ or $f=x^{2}+y^{2}-3 z^{2}$, hence giving a different proof of partial cases (because of the restriction on $m$ ) of theorems of Iwaniec [29] and Duke [14]. Note that a special case of (B) gives an effective equidistribution for the positive definite integral matrices of given determinant. These cases are of special interest since the corresponding symmetric group $\mathbf{H}$ is either compact over the reals or a torus. When $\mathbf{H}$ is semisimple without compact factors over the reals, Corollary 1.10 in its non-effective form, but with no restriction on $m$, is obtained in [20] using Ratner's work on the theory of unipotent flows.

Corollary 1.11. Keep the same assumption as in Corollary 1.10 and set $m_{S}$ to be the product of the finite $p \in S$. For the case (A), we further suppose that $f$ represents 0 over $\mathbb{Q}_{p}$ for at least one $p \in S$. Then there exists $\delta>0$ such that

$$
\#\left\{x \in \mathbf{V}(\mathbb{Z})^{\text {prim }}:\|x\|_{\infty}<T, \quad f(x) \in\langle S\rangle\right\}=v_{T}\left(1+O\left(T^{-\delta}\right)\right)
$$

where the asymptotic $v_{T}$ is given by the following sum over the divisors $m$ of $m_{S}^{d-1}$ :

$$
v_{T}=\sum_{m \mid m_{S}^{d-1}} \widetilde{\operatorname{vol}}\left(\left\{x \in\left(\mathbf{V}_{m}\right)_{S}: \mathrm{H}_{S}(x)<T\right\}\right)
$$

To prove corollaries 1.10 and 1.11, we will apply the effective versions of Theorems 1.1 and 1.4 to $\mathbf{V}_{1}\left(\mathbb{Z}_{S}\right)$. We list more examples in section 15 .

1.3. Guideline. We tried to help the reader in writing "twice" the proofs: In the first half of this paper we concentrate on the main term in the counting and equidistribution statements. In the second half, we follow the same strategy but develop more technical tools to obtain the effective versions of these statements, i.e. to control the error terms.

In section 2, we recall the decay of matrix coefficients for semisimple groups $G$ and its application on a homogeneous space $\Gamma \backslash G$ of finite volume. In section 3, we show the wavefront property for symmetric spaces $H \backslash G$ over local fields and their products. In section 4, we explain how mixing and wavefront properties imply the equidistribution properties of translates of $H$-orbits in $\Gamma \backslash G$ given in Theorem 1.8 . In sections 5 and 6 , we explain how these equidistribution properties for translates of $H$-orbits in $\Gamma \backslash G$ allow us to compare for well-rounded sequences of functions on $H \backslash G$ each sum over a $\Gamma$-orbit with the integral on $H \backslash G$. In section 8 , we give examples of well-rounded sequences and give proofs of Theorem 1.1, Corollary 1.2. Theorem 1.4 and Corollary 1.6.

Starting from section 9, we prove the effective results listed in the introduction. Theorem 1.9 is proved in section 11, and Theorems 1.3, 1.7, Corollaries 1.10 and 1.11, among other effective applications, are proved in section 14. We list more concrete examples in section 15 .

In the appendix 16, we give some general estimates for the volume of balls in the orbits of algebraic groups both over the real and p-adic numbers. 
We remark that in the whole paper the assumption of $\mathbf{H}$ symmetric is used only to obtain the (effective) wave front property for $\mathbf{H}_{S} \backslash \mathbf{G}_{S}$. The methods and the arguments in this paper work equally well for any $K$-subgroup $\mathbf{H}$ with no non-trivial characters satisfying the wave front property.

Acknowledgment The authors would like to thank Alex Gorodnik for helpful conversations.

\section{THE MIXING PROPERTY}

We first recall the Howe-Moore property also called decay of matrix coefficients.

Definition 2.1. A locally compact group $G$ is said to have the Howe-Moore property if, for every unitary representation $(\mathcal{H}, \pi)$ of $G$ containing no non-zero vectors invariant by a normal non-compact subgroup, we have for all $v, w \in \mathcal{H}$,

$$
\lim _{g \rightarrow \infty}\langle\pi(g) v, w\rangle=0 \text {. }
$$

This Howe-Moore property is related to the following mixing property.

Let $G$ be a (unimodular) locally compact group and $\Gamma$ a lattice in $G$, i.e. a discrete subgroup of finite covolume. Let $\mu_{X}$ be a $G$-invariant measure on $X:=\Gamma \backslash G$. The group $G$ acts on $X$ by right-translations.

Definition 2.2. The action of $G$ on $X$ is said to be mixing if for all $\alpha$ and $\beta \in L^{2}(X)$

$$
\lim _{g \rightarrow \infty} \int_{X} \alpha(g x) \beta(x) d \mu_{X}(x)=\mu_{X}(X) \int_{X} \alpha d \mu_{X} \int_{X} \beta d \mu_{X}
$$

The relation between these two definitions is given by the following straightforward proposition.

Definition 2.3. A lattice $\Gamma$ in a locally compact group $G$ is called irreducible if for any non-compact normal subgroup $G^{\prime}$ of $G$, the subgroup $\Gamma G^{\prime}$ is dense in $G$.

Note that this definition is slightly stronger than the usual definition since it excludes lattices contained in a proper subgroup of $G$.

Proposition 2.4. Let $G$ be a locally compact group satisfying the Howe-Moore property and $\Gamma$ an irreducible lattice in $G$. Then the action of $G$ on $\Gamma \backslash G$ is mixing.

Proof. This is well-known. One may assume that $\alpha$ and $\beta$ belong to $\mathcal{H}:=$ $L_{0}^{2}(X)$ of square-integrable functions with zero integral. The $G$-action by righttranslations on $\mathcal{H}$ via $(\pi(g) f)(x)=f(x g)$ is a unitary representation of $G$. The irreducibility hypothesis on $\Gamma$ implies precisely that $\mathcal{H}$ does not contain any nonzero vector invariant by a normal non-compact subgroup of $G$. Hence, by Definition [2.1, the matrix coefficients $\langle\pi(g) \alpha, \beta\rangle$ converge to 0 as $g$ tends to infinity. 
The main example is due to Howe-Moore.

Theorem 2.5. For $i=1, . ., m$, let $k_{i}$ be a local field and $G_{i}$ the group of $k_{i}$ points of a connected semisimple $k_{i}$-group. Then the product $G:=\prod_{i=1}^{m} G_{i}$ has the Howe-Moore property.

In this paper, "local field" means "locally compact field", i.e. a completion of a global field, or, equivalently, a finite extension of $\mathbb{R}, \mathbb{Q}_{p}$ or $\mathbb{F}_{p}((t))$.

Proof. See, for instance, Proposition II.2.3 of [35] or [3].

\section{The WAVEFront PROPERTY}

The wavefront property was introduced by Eskin and McMullen for real symmetric spaces [17]. Let $G$ be a locally compact group and $H$ a closed subgroup of $G$.

Definition 3.1. The group $G$ has the wavefront property in $H \backslash G$ if there exists a Borel subset $F \subset G$ such that $G=H F$ and, for every neighborhood $U$ of $e$ in $G$, there exists a neighborhood $V$ of e in $G$ such that

$$
H V g \subset H g U \text { for all } g \in F \text {. }
$$

This property means roughly that the $g$-translate of a small neighborhood of the base point $z_{0}:=[H]$ in $H \backslash G$ remains near $z_{0} g$ uniformly over $g \in F$.

This section is devoted to proving the following:

Proposition 3.2. Let $k$ be a local field of characteristic not 2, G a connected semisimple k-group, $\sigma$ a k-involution of $\mathbf{G}, G=\mathbf{G}_{k}$ and $H$ a closed subgroup of finite index in the group $G^{\sigma}$ of $\sigma$-fixed points.

Then the group $G$ has the wavefront property on $H \backslash G$.

To prove the above proposition, we need the following two lemmas. A $k$-torus $\mathbf{S}$ of $\mathbf{G}$ is said to be $(k, \sigma)$-split if it is $k$-split and if $\sigma(g)=g^{-1}$ for all $g \in \mathbf{S}$. By a theorem of Helminck and Wang [28, there are only finitely many $H$-conjugacy classes of maximal $(k, \sigma)$-split tori of $\mathbf{G}$. Choose a set $\left\{\mathbf{A}_{i}: 1 \leq i \leq m\right\}$ of representatives of $H$-conjugacy class of maximal $(k, \sigma)$-split tori of $\mathbf{G}$ and set $A=\cup_{i=1}^{m} \mathbf{A}_{i}(k)$.

The following lemma was proved in [17] for $k=\mathbb{R}$, in [4] for all local fields of characteristic not 2 (and independently in [11] when the residual characteristic is not 2).

Lemma 3.3 (Polar decomposition of symmetric spaces). There exists a compact subset $K$ of $G$ such that

$$
G=H A K
$$

The second lemma we need is based on the work of Helminck and Wang. 
Let $\mathbf{A}$ be a maximal $(k, \sigma)$-split torus of $\mathbf{G}$ and $\mathbf{L}$ the centralizer of $\mathbf{A}$ in $\mathbf{G}$. The set of roots $\Phi=\Phi(\mathbf{G}, \mathbf{A})$ for the action of $\mathbf{A}$ on the Lie algebra of $\mathbf{G}$ is a root system. For every positive root system $\Phi^{+} \subset \Phi$, let $\mathbf{N}$ (resp. $\mathbf{N}^{-}$) be the unipotent subgroup of $\mathbf{G}$ generated by the root groups $\mathbf{U}_{\alpha}$ (resp. $\mathbf{U}_{-\alpha}$ ), for $\alpha \in \Phi^{+}$, let $\mathbf{P}:=\mathbf{L N}$ (resp. $\mathbf{P}^{-}:=\mathbf{L} \mathbf{N}^{-}$) and $\mathbf{A}_{k}^{+}$the Weyl Chamber :

$$
\mathbf{A}_{k}^{+}:=\left\{a \in \mathbf{A}_{k}|| \alpha(a) \mid \leq 1 \text {, for all } \alpha \in \Phi^{+}\right\} .
$$

When $\Phi^{+}$vary, the Weyl chambers form a finite covering of $\mathbf{A}_{k}$. Since $\mathbf{P}^{-}=\sigma(\mathbf{P})$, the parabolic $k$-subgroups $\mathbf{P}$ are $\sigma$-split, i.e., the product $\mathbf{H P}$ is open in $\mathbf{G}[28$, Prop. 4.6 and 13.4]. Conversely, any minimal $\sigma$-split parabolic $k$-subgroups of $\mathbf{G}$ containing $\mathbf{A}$ can be constructed in this way for a suitable choice of $\Phi^{+}$.

Lemma 3.4. (1) The multiplication map $m: \mathbf{H}_{k} \times \mathbf{P}_{k} \rightarrow \mathbf{G}_{k}$ is an open map.

(2) There exists a basis of compact neighborhoods $W$ of e in $\mathbf{P}_{k}$ such that

$$
a^{-1} W a \subset W \quad \text { for all } a \in \mathbf{A}_{k}^{+} .
$$

(3) For every neighborhood $U$ of e in $G$, there exists a neighborhood $V$ of $e$ in G such that

$$
H V a \subset H a U \quad \text { for all } a \in \mathbf{A}_{k}^{+} .
$$

Proof. (1) When char $(k)=0$, it follows from the fact that Lie algebras of $\mathbf{P}_{k}$ and $\mathbf{H}_{k}$ generate the Lie algebra of $\mathbf{G}_{k}$ as a vector space. For a characteristic free argument, see [28] or Proposition I.2.5.4 in [35].

(2) When $\operatorname{char}(k)=0$, note that the action of $\mathbf{A}_{k}^{+}$on the Lie algebra $\operatorname{Lie}\left(\mathbf{P}_{k}\right)$ gives a family of commuting semisimple linear maps $\mathrm{Ad}(a)$ whose eigenvalues have bounded above by 1 in their absolute values. It follows that there exists a basis of compact neighborhoods $W_{0}$ of 0 in $\operatorname{Lie}\left(\mathbf{P}_{k}\right)$ which are invariant by all $\operatorname{Ad}(a), a \in \mathbf{A}_{k}^{+}$. It suffices to set $W=\exp \left(W_{0}\right)$.

It is easy to adapt this argument in positive characteristic case; write $\mathbf{P}_{k}=$ $\mathbf{L}_{k} \mathbf{N}_{k}$, and note that $\mathbf{L}_{k}$ contains a $\mathbf{A}_{k}$-invariant compact open subgroup. Now considering the linear group $\mathbf{N}_{k}$ as a group of upper triangular matrices in a suitable basis where elements of $\mathbf{A}_{k}^{+}$are diagonals with increasing coefficients in absolute value, we can find a basis of compact neighborhoods $W$ as desired.

(3) Choose $W$ as in (2) small enough so that $W \subset U$ and choose any neighborhood $V$ of $e$ in $G$ contained in $H W$. We then have $H V a \subset H W a \subset H a U$, as required.

Proof of Proposition 3.2. We will prove that $G$ has the wavefront property on $H \backslash G$ with the subset $F=A K$ defined in Lemma 3.3. By Lemma 3.3, it suffices to show that for every neighborhood $U$ of e in $G$, there exists a neighborhood $V$ of $e$ in $G$ such that $H V g \subset H g U$ for all $g \in A K$

Recall $A=\cup_{i=1}^{m} \mathbf{A}_{i}(k)$ where $\mathbf{A}_{i}$ is a maximal $(k, \sigma)$-split torus of $G$. Fix $i$ and a positive Weyl chamber $C$ of $\mathbf{A}_{i}(k)$. 
Since $K$ is a compact set, there exists a neighborhood $U_{0}$ of $e$ in $G$ such that $k^{-1} U_{0} k$ is contained in $U$ for all $k$ in $K$. By Lemma 3.4.(3), there exists a neighborhood $V_{C}$ of $e$ in $G$ such that $V_{C} a \subset H a U_{0}$ for all $a \in C$.

Now set $V:=\cap_{C} V_{C}$ where the intersection is taken over all (finitely many) positive Weyl chambers of $\mathbf{A}_{i}(k), 1 \leq i \leq m$. Then for $g=a k \in\left(\cup_{C} C\right) K=A K$ with $k \in K$ and $a \in C$, we have

$$
H V g \subset H V_{C} a k \subset H a U_{0} k \subset H a k U=H g U .
$$

This finishes the proof.

In section 8, we will use this wavefront property in the product situation, owing to the following straightforward proposition.

Proposition 3.5. For $i=1, \ldots, m$, let $G_{i}$ be a locally compact group, $H_{i} \subset G_{i}$ a closed subgroup, $G:=\prod_{i=1}^{m} G_{i}$ and $H:=\prod_{i=1}^{m} H_{i}$. If $G_{i}$ has the wavefront property on $H_{i} \backslash G_{i}$ for each $1 \leq i \leq m$, then $G$ has the wavefront property on $H \backslash G$.

The following theorem is an immediate consequence of Propositions $2.4,2.5$, 3.2 and 3.5 .

Theorem 3.6. For $i=1, . ., m$, let $k_{i}$ be a local field, $G_{i}$ the group of $k_{i}$-points of a semisimple $k_{i}$-group, $\sigma_{i}$ an involution of $G_{i}$ defined over $k_{i}, G_{i}^{\sigma_{i}}$ its group of fixed points and $H_{i}$ a closed subgroup of finite index of $G_{i}^{\sigma_{i}}$. Let $G=\prod_{i=1}^{m} G_{i}$ and $H:=\prod_{i=1}^{m} H_{i}$.

Then the group $G$ has the wavefront property on $H \backslash G$.

Moreover, for any irreducible lattice $\Gamma$ in $G$, the action of $G$ on $\Gamma \backslash G$ is mixing.

Note that this theorem provides many natural examples of triples $(G, H, \Gamma)$ which satisfy the hypothesis of the propositions 4.1, 5.3 and 6.2 .

\section{Equidistribution of translates of $H$-ORBits}

In this section, let $G$ be a locally compact group, $H \subset G$ a closed subgroup, $\Gamma \subset G$ a lattice such that $\Gamma_{H}:=\Gamma \cap H$ is a lattice in $H$. Set $X=\Gamma \backslash G$ and $Y=\Gamma_{H} \backslash H$. Let $\mu_{X}$ and $\mu_{Y}$ be invariant measures on $X$ and $Y$ respectively.

Proposition 4.1. Suppose that the action of $G$ on $X$ is mixing and that $G$ has the wavefront property on $H \backslash G$. Then the translates $Y g$ become equidistributed in $X$, as $g \rightarrow \infty$ in $H \backslash G$.

This means that as the image of $g$ in $H \backslash G$ leaves every compact subsets, the sequence of probability measures $\frac{1}{\mu_{Y}(Y)} g_{*} \mu_{Y}$ weakly converges to $\frac{1}{\mu_{X}(X)} \mu_{X}$, i.e., for any $\psi \in C_{c}(X)$, we have

$$
\frac{1}{\mu_{Y}(Y)} \int_{Y} \psi(y g) d \mu_{Y}(y) \rightarrow \frac{1}{\mu_{X}(X)} \int_{X} \psi d \mu_{X} .
$$


Proof. The following proof is adapted from [17]; we point out that the case when $Y$ is non-compact requires a bit more care, which was not addressed in [17]. Since $G=H F$, we may assume that $g$ belongs to the subset $F$ in Definition 3.1. We assume, without loss of generalities, that $\mu_{X}$ and $\mu_{Y}$ are probability measures. Let $\psi \in C_{c}(X)$. Fix $\varepsilon>0$. By the uniform continuity of $\psi$ there exists a neighborhood $U$ of $e$ in $G$ such that

$$
|\psi(x u)-\psi(x)|<\varepsilon \quad \text { for all } u \in U \text { and } x \in X \text {. }
$$

By the wavefront property of $G$ on $H \backslash G$, there exists a compact neighborhood $V \subset U$ of $e$ in $G$ such that

$$
V g \subset H g U \text { for all } g \in F
$$

Choose a compact subset $Y_{\epsilon} \subset Y$ of measure at least $\mu_{Y}\left(Y_{\epsilon}\right) \geq 1-\varepsilon$. Choose a Borel subset $W \subset V$ in $G$ transversal to $H$, i.e., a subset $W$ of $G$ such that the multiplication $m: H \times W \rightarrow G$ is injective with the image $H W$ being an open neighborhood of $e$ in $G$. Using the compactness of $Y_{\epsilon}$ and the discreteness of $\Gamma$, we may assume that the image of $W$ in $H \backslash G$ is small enough so that the multiplication $m: Y_{\epsilon} \times W \rightarrow Y_{\epsilon} W$ is a bijection 1 onto its image $Y_{\varepsilon} W \subset X$.

Let $\mu_{W}$ be the measure on $W$ such that $\mu_{X}=\mu_{Y} \mu_{W}$ locally.

Setting

$$
I_{g}:=\int_{Y} \psi(y g) d \mu_{Y}(y)
$$

we need to show that

$$
I_{g} \rightarrow \int_{X} \psi d \mu_{X} \quad \text { as } g \in F \text { goes to infinity in } G .
$$

For simplicity, set

$$
J_{g}=\frac{1}{\mu_{W}(W)} \int_{Y \times W} \psi(y w g) d \mu_{Y}(y) d \mu_{W}(w) \text { and } K_{g}=\frac{1}{\mu_{W}(W)} \int_{Y_{\varepsilon} W} \psi(x g) d \mu_{X}(x) .
$$

Roughly speaking, we will argue that $I_{g}$ is close to $J_{g}$ as a consequence of the wavefront property, $J_{g}$ is close to $K_{g}$ since the volume of $Y-Y_{\varepsilon}$ is small, and finally $K_{g}$ is close to the average of $\psi$ for large $g$ because of the mixing property.

\footnotetext{
${ }^{1}$ When $Y$ is compact, one can choose the transversal $W$ such that the map $Y \times W \rightarrow Y W$ is bijective onto an open subset of $X$. When $Y$ is not compact, such a transversal does not always exist. Here is an example: let $G$ be the orthogonal group of the quadratic form $x^{2}+y^{2}+z^{2}-t^{2}$ on $\mathbb{R}^{4}, v_{0}=(1,0,0,0), v_{1}=(1,0,2,2), \Gamma=G_{\mathbb{Z}}$ and $H$ the stabilizer of the point $v_{0}$. One checks easily that (a) $v_{1}=\gamma v_{0}$ for some $\gamma \in \Gamma$ and that (b) $v_{0}$ is a limit of elements $v_{1} h_{n}$ of the $H$-orbit of $v_{1}$. Hence there exists a sequence $g_{n}$ converging to $e$ in $G$ such that $H g_{n} \cap \gamma H \neq \emptyset$. To check (a), take $\gamma=\left(\begin{array}{cccc}1 & 0 & 2 & 2 \\ 0 & 1 & 0 & 0 \\ 2 & 0 & 1 & 2 \\ 2 & 0 & 2 & 3\end{array}\right)$. For (b), take $h_{n}=\left(\begin{array}{cccc}1 & 0 & 0 & 0 \\ 0 & 1 & 0 & 0 \\ 0 & 0 & \cosh n & -\sinh n \\ 0 & 0 & -\sinh n & \cosh n\end{array}\right)$.
} 
By (4.4), for each $w \in W$ and $g \in F$, we have $w g=h_{g, w} g u$ for some $h_{g, w} \in H$. Hence

$$
\begin{aligned}
\left|I_{g}-\int_{Y} \psi(y w g) d \mu_{Y}(y)\right| & =\left|\int_{Y} \psi(y g) d \mu_{Y}(y)-\int_{Y} \psi\left(y h_{g, w} g u\right) d \mu_{Y}(y)\right| \\
& =\left|\int_{Y}(\psi(y g)-\psi(y g u)) d \mu_{Y}(y)\right| \leq \varepsilon \quad \text { by (4.3) } .
\end{aligned}
$$

Therefore we have

$$
\left|I_{g}-J_{g}\right| \leq \varepsilon
$$

By the choice of $W$, we have

$$
K_{g}=\frac{1}{\mu_{W}(W)} \int_{W} \int_{Y_{\varepsilon}} \psi(y w g) d \mu_{Y}(y) d \mu_{W}(w)
$$

and hence

$$
\left|J_{g}-K_{g}\right| \leq 2 \mu_{Y}\left(Y-Y_{\varepsilon}\right)\|\psi\|_{\infty} \leq 2\|\psi\|_{\infty} \varepsilon .
$$

Since $K_{g}=\frac{1}{\mu_{W}(W)} \int_{X} \psi(x g) \mathbf{1}_{Y_{\varepsilon} W}(x) d \mu_{X}(x)$ where $\mathbf{1}_{Y_{\varepsilon} W}$ is the characteristic function of $W Y_{\varepsilon}$, the mixing property of $G$ on $\Gamma \backslash G$ says that $K_{g}$ converges to $\mu_{Y}\left(Y_{\varepsilon}\right) \int_{X} \psi d \mu_{X}$ as $g \rightarrow \infty$ in $F$. Hence for $g \in F$ large enough we have,

$$
\left|K_{g}-\int_{X} \psi d \mu_{X}\right| \leq \varepsilon+\mu_{Y}\left(Y-Y_{\varepsilon}\right) \int_{X} \psi d \mu_{X} \leq\left(1+\|\psi\|_{\infty}\right) \varepsilon .
$$

Putting this together, we get

$$
\begin{aligned}
\left|I_{g}-\int_{X} \psi d \mu_{X}\right| & \leq\left|I_{g}-J_{g}\right|+\left|J_{g}-K_{g}\right|+\left|K_{g}-\int_{X} \psi d \mu_{X}\right| \\
& \leq\left(2+3\|\psi\|_{\infty}\right) \varepsilon .
\end{aligned}
$$

Since $\varepsilon>0$ is arbitrary, this shows the claim.

Using Theorem 3.6, we obtain:

Corollary 4.6. Let $G, H, \Gamma$ be as in Theorem [3.6. Then the translates $Y g:=$ $\Gamma_{H} \backslash H g$ become equidistributed in $X:=\Gamma \backslash G$ as $g \rightarrow \infty$ in $H \backslash G$.

\section{SUMS AND INTEGRALS}

Let $G$ be a locally compact group, $H \subset G$ a closed subgroup, $\Gamma \subset G$ a lattice such that $\Gamma_{H}:=\Gamma \cap H$ is a lattice in $H$. Let $x_{0}:=[\Gamma]$ be the base point in $X:=\Gamma \backslash G, Y=x_{0} H$ and $z_{0}:=[H]$ be the base point in $Z:=H \backslash G$. We note that $z_{0} \Gamma$ is a discrete subset of $Z$. There exist $G$-invariant measures $\mu_{X}, \mu_{Y}$ and $\mu_{Z}$ on $X, Y$ and $Z$. We normalize them so that $\mu_{X}=\mu_{Z} \mu_{Y}$ locally.

For a given sequence of non-negative functions $\varphi_{n}$ on $Z$ with compact support, we define a function $F_{n}$ on $X$ so that, for $x=x_{0} g, F_{n}(x)$ is the sum of $\varphi_{n}$ over the discrete orbit $z_{0} \Gamma g$ : 


$$
F_{n}(x):=\sum_{\gamma \in \Gamma_{H} \backslash \Gamma} \varphi_{n}\left(z_{0} \gamma g\right) \quad \text { for } x=\Gamma g .
$$

We would like to compare the values of $F_{n}$ with the space average over $Z$ :

$$
I_{n}:=\frac{\mu_{Y}(Y)}{\mu_{X}(X)} \int_{Z} \varphi_{n}(z) d \mu_{Z}(z)
$$

We remark that this normalized integral $I_{n}$ does not depend on the choices of measures.

The following proposition 5.3 says that the sum $F_{n}$ is asymptotic to the normalized integral $I_{n}$, at least weakly.

Proposition 5.3. Suppose that the translates $Y g$ become equidistributed in $X$ as $g \rightarrow \infty$ in $Z$. Then for any sequence of non-negative functions $\varphi_{n}$ on $Z$ with compact support such that $\max _{n}\left\|\varphi_{n}\right\|_{\infty}<\infty$ and $\lim _{n \rightarrow \infty} \int_{Z} \varphi_{n} d \mu_{Z}=\infty$, the ratios $F_{n}(x) / I_{n}$ converge weakly to 1 as $n \rightarrow \infty$.

This means that, for all $\alpha \in C_{c}(X)$,

$$
\lim _{n \rightarrow \infty} \frac{1}{I_{n}} \int_{X} F_{n}(x) \alpha(x) d \mu_{X}(x)=\int_{X} \alpha(x) d \mu_{X}(x) .
$$

Proof. Using transitivity properties for invariant integration on homogeneous spaces, we obtain that for all $\alpha \in C_{c}(X)$,

$$
\begin{aligned}
\int_{\Gamma \backslash G} F_{n} \alpha d \mu_{\Gamma \backslash G} & =\int_{\Gamma \backslash G} \sum_{\gamma \in \Gamma_{H} \backslash \Gamma} \varphi_{n}(H \gamma g) \alpha(\Gamma g) d \mu_{\Gamma \backslash G}(\Gamma g) \\
& =\int_{G_{H} \backslash \Gamma} \varphi_{n}(H g) \alpha(\Gamma g) d \mu_{\Gamma \backslash G_{H}}\left(\Gamma_{H} g\right) \\
& =\int_{H \backslash G} \int_{\Gamma_{H} \backslash H} \varphi_{n}(H g) \alpha\left(\Gamma_{H} h g\right) d \mu_{\Gamma_{H} \backslash H}\left(\Gamma_{H} h\right) d \mu_{H \backslash G}(H g) \\
& =\int_{H \backslash G} \varphi_{n}(z) \beta(z) d \mu_{H \backslash G}(z)
\end{aligned}
$$

where $\beta$ is the function on $Z$ given by,

$$
\begin{aligned}
\beta(H g) & =\int_{\Gamma_{H} \backslash H} \alpha\left(\Gamma_{H} h g\right) d \mu_{\Gamma_{H} \backslash H}\left(\Gamma_{H} h\right) \\
& =\int_{Y} \alpha(y g) d \mu_{Y}(y) .
\end{aligned}
$$


By assumption, we have

$$
\lim _{z \rightarrow \infty} \beta(z)=\frac{\mu_{Y}(Y)}{\mu_{X}(X)} \int_{X} \alpha(x) d \mu_{X}(x) .
$$

Since $I_{n}=\int_{Z} \varphi_{n} \rightarrow \infty$ and $\varphi_{n}$ are uniformly bounded, by the dominated convergence theorem

$$
\lim _{n \rightarrow \infty} \frac{1}{I_{n}} \int_{Z} \varphi_{n}(z) \beta(z) d \mu_{Z}(z)=\int_{X} \alpha d \mu_{X}
$$

Hence we obtain the equality (5.4).

\section{Counting And EQuidistribution}

We will now improve the weak convergence in proposition 5.3 to the pointwise convergence of the functions $F_{n}$. This requires some hypothesis on the sequence of functions $\varphi_{n}$ which will be called well-roundedness. We keep the notations of section 5 .

Definition 6.1. A sequence or a family of non-negative integrable functions $\varphi_{n}$ of $Z$ with compact support is said to be well-rounded if for any $\varepsilon>0$, there exists a neighborhood $U$ of $e$ in $G$, such that the following holds for all $n$.

$$
(1-\varepsilon) \int_{Z}\left(\sup _{u \in U} \varphi_{n}(z u)\right) d \mu_{Z}(z) \leq \int_{Z} \varphi_{n} d \mu_{Z} \leq(1+\varepsilon) \int_{Z}\left(\inf _{u \in U} \varphi_{n}(z u)\right) d \mu_{Z}(z) \text {. }
$$

$A$ sequence of subsets $B_{n}$ of $Z$ is said to be well-rounded if the sequence $\mathbf{1}_{B_{n}}$ is well-rounded.

Sometimes we will apply the above definition to a continuous family $\left\{\varphi_{T}\right\}$ of functions or subsets, whose meaning should be clear.

Recall that we want to compare the orbital sum $F_{n}\left(x_{0}\right)=\sum_{\gamma \in \Gamma / \Gamma_{H}} \varphi_{n}\left(\gamma z_{0}\right)$ with the average $I_{n}=\frac{\mu_{Y}(Y)}{\mu_{X}(X)} \int_{Z} \varphi_{n}(z) d \mu_{Z}(z)$.

Proposition 6.2. Keep the notations and hypothesis of Proposition 5.3, and assume that the sequence $\varphi_{n}$ is well-rounded. Then,

$$
F_{n}\left(x_{0}\right) \sim I_{n} \text { as } n \rightarrow \infty .
$$

The notation $a_{n} \sim b_{n}$ means that the ratio of $a_{n}$ and $b_{n}$ tends to 1 as $n \rightarrow \infty$.

Proof. Once again, we may normalize the measures so that $\mu_{X}(X)=\mu_{Y}(Y)=1$. Fix $\varepsilon>0$ and let $U$ be a neighborhood of $e$ in $G$ given by Definition 6.1. We introduce the functions $\varphi_{n}^{ \pm}$on $Z$ defined by

$$
\varphi_{n}^{+}(z):=\sup _{u \in U} \varphi_{n}\left(z u^{-1}\right) \text { and } \varphi_{n}^{-}(z):=\inf _{u \in U} \varphi_{n}\left(z u^{-1}\right)
$$

and their integrals $I_{n}^{ \pm}:=\int_{Z} \varphi_{n}^{ \pm} d \mu_{Z}$. Note that for each $n$,

$$
(1-\varepsilon) I_{n}^{+} \leq I_{n} \leq(1+\varepsilon) I_{n}^{-} \text {. }
$$


We also introduce the functions $F_{n}^{ \pm}$on $X$ :

$$
F_{n}^{ \pm}(x)=\sum_{\gamma \in \Gamma_{H} \backslash \Gamma} \varphi_{n}^{ \pm}\left(z_{0} \gamma g\right) \quad \text { for } x=\Gamma g .
$$

It is easy to check that, for all $u \in U$ and $x \in X$

$$
F_{n}^{-}(u x) \leq F_{n}(x) \leq F_{n}^{+}(u x) .
$$

Choose a non-negative continuous function $\alpha$ on $X$ with $\int_{X} \alpha=1$ and with support included in $x_{0} U$ so that the following holds for all $n$ :

$$
\int_{X} \alpha F_{n}^{-} d \mu_{X} \leq F_{n}\left(x_{0}\right) \leq \int_{X} \alpha F_{n}^{+} d \mu_{X}
$$

Applying Proposition 5.3 to the sequences of functions $\varphi_{n}^{ \pm}$, we obtain, for all $n$ large,

$$
(1-\varepsilon) I_{n}^{-} \leq F_{n}\left(x_{0}\right) \leq(1+\varepsilon) I_{n}^{+} .
$$

Using the estimations (6.3) and (6.4), every cluster value of the sequence of ratios $F_{n}\left(x_{0}\right) / I_{n}$ is within the interval $\left[\frac{1-\varepsilon}{1+\varepsilon}, \frac{1+\varepsilon}{1-\varepsilon}\right]$. Hence this sequence converges to 1 .

\section{WELL-ROUNDEDNESS}

In this section, we provide explicit examples of well-rounded sequences $\varphi_{n}$ in order to apply Proposition 6.2. We start with an observation that the product of well-rounded sequences is again well-rounded.

Example 7.1. For each $i=1, \ldots, m$, let $G_{i}$ be a locally compact group, $H_{i} \subset G_{i}$ a closed subgroup and $\varphi_{i, n}$ be a well-rounded sequence of functions on $Z_{i}:=H_{i} \backslash G_{i}$. Let $G:=\prod_{i=1}^{m} G_{i}, H:=\prod_{i=1}^{m} H_{i}$ and $Z:=\prod_{i=1}^{m} Z_{i}$. Then the sequence $\varphi_{n}$ defined by

$$
\varphi_{n}\left(z_{1}, \ldots, z_{m}\right)=\prod_{1 \leq i \leq m} \varphi_{i, n}\left(z_{i}\right)
$$

is well-rounded.

Proof. Fix $\varepsilon>0$. Let $U_{i}$ be a neighborhood of $e$ in $G_{i}$ such that the functions $\varphi_{i, n}^{ \pm}$ on $Z_{i}$ defined by $\varphi_{i, n}^{+}\left(z_{i}\right)=\sup _{u_{i} \in U_{i}} \varphi_{i, n}\left(z_{i} u_{i}\right)$ and $\varphi_{i, n}^{-}\left(z_{i}\right)=\inf _{u_{i} \in U_{i}} \varphi_{i, n}\left(z_{i} u_{i}\right)$ satisfy

$$
(1-\varepsilon) \int_{Z_{i}} \varphi_{i, n}^{+} \leq \int_{Z_{i}} \varphi_{i, n} \leq(1+\varepsilon) \int_{Z_{i}} \varphi_{i, n}^{-} .
$$

Let $U:=\prod U_{i}$, and $\varphi_{n}^{ \pm}$be the functions on $Z$ defined by

$$
\varphi_{n}^{+}(z)=\sup _{u \in U} \varphi_{n}(z u) \quad \text { and } \quad \varphi_{n}^{-}(z)=\inf _{u \in U} \varphi_{n}(z u)
$$

so that $\varphi_{n}^{ \pm}=\prod \varphi_{i, n}^{ \pm}$and

$$
(1-\varepsilon)^{m} \int_{Z} \varphi_{n}^{+} \leq \int_{Z} \varphi_{n} \leq(1+\varepsilon)^{m} \int_{Z} \varphi_{n}^{-} .
$$

Hence the sequence $\varphi_{n}$ is well-rounded. 
The next example deals with the constant sequences. It will be used both for the archimedean and the non-archimedean factors.

Example 7.2. Let $G$ be a locally compact group, $H$ a closed subgroup of $G$, $Z=H \backslash G$, and $\varphi \in C_{c}(Z)$ with $\varphi \geq 0$ and $\varphi \neq 0$. Then the constant sequence $\varphi_{n}=\varphi$ is well-rounded.

Proof. Use the uniform continuity of $\varphi$ and the compactness of its support.

The following example deals with the archimedean factors.

Example 7.3. Let $G$ be a real semisimple Lie group with finitely many connected components, $V$ a finite dimensional representation of $G, Z$ a closed $G$-orbit in $V$ with an invariant measure $\mu$ and $\|$.$\| an euclidean norm on V$. Then the family of balls $B_{T}:=\{z \in Z \mid\|z\| \leq T\}, T \gg 1$ is well-rounded.

Proof. By Corollary 16.3. a of the appendix, we have

$$
\mu\left(B_{T}\right) \sim_{T} c T^{a}(\log T)^{b}
$$

for some $a \in \mathbb{Q}_{\geq 0}, b \in \mathbb{Z}_{\geq 0}$ and $c>0$. It is easy to deduce the claim from the above asymptotic using the assumption that the action of $G$ is linear on $Z$.

As for the non-archimedean factors, we have:

Example 7.4. Let $k$ be a non-archimedean local field, $G$ the group of $k$-points of a connected semisimple k-group, $\rho: G \rightarrow G L(V)$ a representation of $G$ defined over $k, Z$ a closed $G$-orbit in $V$ with an invariant measure and $\|\cdot\|$ a norm on $V$. Then, both the family of non empty balls $B_{T}:=\{z \in Z \mid\|z\| \leq T\}$, and the family of non-empty spheres $S_{T}:=\{z \in Z \mid\|z\|=T\}$, are well-rounded.

Proof. Since the action of $G$ on $V$ is linear, the stabilizer in $G$ of the norm is a compact open subgroup of $G$. Hence this example is a special case of the following easy assertion.

Example 7.5. Let $G$ be a locally compact (unimodular) group, $H$ a closed (unimodular) subgroup of $G, Z=H \backslash G$ and $U$ a compact open subgroup of $G$. Then any sequence $\varphi_{n}$ of non-negative $U$-invariant $L^{1}$-functions on $Z$ is well-rounded.

As the last example, we will show that a sequence of the height balls is well rounded. We will need the following basic lemma.

Lemma 7.6. Let $I$ be a finite set. For each $i \in I$, let $\tau_{i}>1$ and $\lambda_{i}>0$ be given. Let $\lambda: \mathbb{N}^{I} \rightarrow \mathbb{R}^{+}$be given by $\lambda(m)=\sum_{i \in I} \lambda_{i} m_{i}$ for $m=\left(m_{i}\right)$, and $P: \mathbb{N}^{I} \rightarrow \mathbb{R}^{+}$ a function given by $P(m)=\prod_{i \in I} P_{i}\left(m_{i}\right)$ where $P_{i}$ is a real-valued function of a variable $x$ given by a polynomial expression in $\left(x, \tau_{i}^{x}\right)$ and which is positive on $\mathbb{N}$. Then we have

$$
w_{t+1}=O\left(w_{t}\right) \quad \text { for } t \text { large }
$$


where

$$
w_{t}:=\sum_{\left\{m \in \mathbb{N}^{I}, \lambda(m) \leq t\right\}} P(m) .
$$

Proof. Since each $P_{i}$ is positive on $\mathbb{N}$, there exists $C>0$ such that for $i \in I$ and $n \in \mathbb{N}$, one has

$$
P_{i}(n+1) \leq C P_{i}(n) .
$$

Hence for each $m \in \mathbb{N}^{I}$ and each $e$ in the basis $E$ of $\mathbb{N}^{I}$, one has

$$
P(m+e) \leq C P(m) .
$$

Setting $t_{0}:=\min _{i} \lambda_{i}=\min _{e \in E} \lambda(e)$, one gets

$$
w_{t+t_{0}} \leq \sum_{e \in E} \sum_{\lambda(m) \leq t} P(m+e) \leq r C w_{t} .
$$

for $r=|I|$. Hence we conclude that $w_{t+1} \leq(r C)^{k} w_{t}$ with $k=\frac{1}{t_{0}}+1$.

Remark 7.7. One can improve the conclusion of Lemma 7.6, there exist $a \geq 0$, $b \in \mathbb{Z}_{\geq 0}$ and $c_{1}, c_{2}>0$ such that, for $t$ large,

$$
c_{1} e^{a t} t^{b} \leq w_{t} \leq c_{2} e^{a t} t^{b}
$$

Moreover, setting $C_{i} \tau_{i}^{d_{i} x} x^{b_{i}}$ for the dominant term of $P_{i}(x)$, the exponents $a$ and $b$ are given respectively by $e^{a}=\max _{i \in I} \tau_{i}^{d_{i} / \lambda_{i}}$ and $b$ is given by $b=\sum_{i}\left(b_{i}+1\right)-1$ where the sum is taken over all $i$ such that $e^{a}=\tau_{i}^{d_{i} / \lambda_{i}}$. The proof is a straightforward induction on $|I|$. Here is a sketch: one may assume that, for all $i, P_{i}(x)=C_{i} e^{a_{i} x} x^{b_{i}}$ and $\lambda_{i}=1$. One fixes $i_{0} \in I$, set $\check{I}:=I-\left\{i_{0}\right\}$ and writes $w_{t}:=\sum_{1 \leq n \leq t} P_{i_{0}}(n) \check{w}_{t-n}$ where, by induction hypothesis, $\check{w}_{t}$ satisfies a similar estimation, as $t \rightarrow \infty$ : $\check{c}_{1} e^{\check{a} t} t^{\check{b}} \leq \check{w}_{t} \leq \check{c}_{2} e^{\check{a} t} t^{\check{b}}$ for some $\check{a} \geq 0, \check{b} \in \mathbb{Z}_{\geq 0}$ and $\check{c}_{1}, \check{c}_{2}>0$. From that, one gets the required estimation for $w_{t}$.

For the rest of this section, let $I$ be a finite set. For each $i \in I$, let $k_{i}$ be a local field of characteristic $0, G_{i}$ the group of $k_{i}$-points of an algebraic $k_{i}$-group, $V_{i}$ an algebraic representation of $G_{i}$, and $Z_{i} \subset V_{i}$ a non-zero closed $G_{i}$-orbit with an invariant measure $\mu_{i}$.

We set

$$
G:=\prod_{i \in I} G_{i}, Z:=\prod_{i \in I} Z_{i}, \mu:=\otimes_{i \in I} \mu_{i} .
$$

Let $I_{\infty} \subset I$ be the set of indices with $k_{i}$ archimedean, and $I_{f}:=I \backslash I_{\infty}$. The partition $I=I_{\infty} \sqcup I_{f}$ induces decompositions $G=G_{\infty} \times G_{f}$ of the group, $Z=Z_{\infty} \times Z_{f}$ of the orbit, and $\mu=\mu_{\infty} \otimes \mu_{f}$ of the invariant measure. 
Let $\|\cdot\|_{i}$ be a norm on $V_{i}$. We assume that $\|\cdot\|_{i}$ is euclidean if $i \in I_{\infty}$ and a $\max$ norm otherwise. These norms define a height function $h: Z \rightarrow \mathbb{R}^{+}$

$$
h(z)=\prod_{i \in I}\left\|z_{i}\right\|_{i} .
$$

Since each $Z_{i}$ is a closed non-zero subset in $V_{i}$, we have $\min _{z \in Z_{i}}\|z\|_{i}>0$ and hence $h$ is a proper function on $Z$. We can also write $h=h_{\infty} \otimes h_{f}$ where $h_{\infty}:=\prod_{i \in I_{\infty}}\|\cdot\|_{i}$ and $h_{f}:=\prod_{i \in I_{f}}\|\cdot\|_{i}$.

Set

$$
\begin{gathered}
b_{T}:=\left\{z \in Z_{\infty} \mid h_{\infty}(z) \leq T\right\}, \beta_{T}:=\left\{z \in Z_{f} \mid h_{f}(z) \leq T\right\}, \\
V_{T}:=\mu\left(B_{T}\right), v_{T}=\mu_{\infty}\left(b_{T}\right) \text { and } w_{T}=\mu_{f}\left(\beta_{T}\right) .
\end{gathered}
$$

Lemma 7.8. Assume that $h_{\infty}$ is not constant on $Z_{\infty}$.

(1) There exist $a \in \mathbb{Q}_{\geq 0}, b \in \mathbb{Z}_{\geq 0}$, and $c>0$ such that as $T \rightarrow \infty$,

$$
v_{T} \sim c T^{a}(\log T)^{b} \quad \text { and } \quad \frac{d}{d T} v_{T} \sim c \frac{d}{d T}\left(T^{a}(\log T)^{b}\right) .
$$

(2) There exist constants $\kappa>0$ and $C_{1}>0$ such that for all $\left.\varepsilon \in\right] 0,1[$ and all $T \geq 0$

$$
v_{(1+\varepsilon) T}-v_{T} \leq C_{1}\left(v_{T}+1\right) \varepsilon^{\kappa} .
$$

(3) For $T$ large, one has $w_{2 T}=O\left(w_{T}\right)$.

(4) There exist $\kappa>0$ such that uniformly for $T$ large and $\varepsilon \in] 0,1$,

$$
V_{(1+\varepsilon) T}=\left(1+O\left(\varepsilon^{\kappa}\right)\right) V_{T} .
$$

Proof. (1): Apply Proposition 16.2 of the appendix to the regular function $F:=$ $h_{\infty}^{2}$ on the orbit $Z_{\infty}$. Note that since $v_{T}$ is an increasing function of $T$, one has $a \geq 0$. Moreover, note that, when $a=b=0$, the orbit is of finite volume and hence compact.

(2): First note that, since $h_{\infty}$ is not constant on $Z_{\infty}$, the function $v_{T}$ is continuous.

When $T$ is large, we use (1) to get the following bound $v_{(1+\varepsilon) T}-v_{T}=O\left(\varepsilon v_{T}\right)$ which is uniform in $\varepsilon \in] 0,1[$.

When $T$ is bounded, we use the fact that the function $v_{T}$ is differentiable except at the critical values $\tau$ of $h_{\infty}$. Since $h_{\infty}^{2}$ is a regular function, there are only finitely many such critical values $\tau$. Around these points, there exists a constant $\kappa, 0<\kappa<1$ such that, for $\varepsilon>0$ small, one has the following bound for the derivative:

$$
v_{\tau \pm \varepsilon}^{\prime}=O\left(\varepsilon^{\kappa-1}\right) .
$$

This assertion is a consequence of Theorem 16.1 of the appendix. More precisely, set $f:= \pm\left(h_{\infty}-\tau\right)$. Since $Z_{\infty}$ is smooth, one can choose $\varepsilon_{0}>0$ and an open covering $U_{j}$ of $f^{-1}(]-\varepsilon_{0}, \varepsilon_{0}[)$ by open sets bianalytically homeomorphic to balls. A partition of unity gives us $\mathrm{C}^{\infty}$ functions $\varphi_{j}$ with compact support in $U_{j}$ such 
that $\sum_{j} \varphi_{j}=1$ on $f^{-1}(]-\varepsilon_{0} / 2, \varepsilon_{0} / 2[)$. We simply apply Theorem 16.1 to these functions $f$ and $\varphi_{j}$ to get (7.9).

Integrating $v_{t}^{\prime}$ on the interval $[T,(1+\varepsilon) T]$, and using (17.9) near the critical values in this interval, one gets, uniformly for $\varepsilon$ small and $T$ bounded,

$$
v_{(1+\varepsilon) T}-v_{T}=O\left(\varepsilon^{\kappa}\right) .
$$

Putting these together proves the claim.

(3): We will assume, as we may, that $\inf _{z \in Z_{i}}\|z\|_{i} \geq 1$ for each $i \in I_{f}$. For any $\left|I_{f}\right|$-tuple $m=\left(m_{i}\right)_{i \in I_{f}} \in \mathbb{N}^{I_{f}}$, we set

$$
S(m)=\prod_{i \in I_{f}} S_{i}\left(m_{i}\right) \quad \text { where } \quad S_{i}\left(m_{i}\right):=\left\{z \in Z_{i}:\|z\|_{i}=m_{i}\right\} .
$$

Letting $\omega_{m}:=\mu_{f}(S(m))$ and $\pi_{m}:=\prod_{i} m_{i}$, one has

$$
w_{T}=\sum_{\left\{m \in \mathcal{M}, \pi_{m} \leq T\right\}} \omega_{m}
$$

where $\mathcal{M} \subset \mathbb{N}^{I_{f}}$ consists of $m \in \mathbb{N}^{I_{f}}$ with non-empty $S(m)$. The main point of the proof is to use the formula for $\omega_{m}$ given by Theorem 16.6 of the appendix. According to this formula, there is a finite partition of $\mathcal{M}$ in finitely many pieces $\mathcal{M}_{\alpha}$ such that

- each piece $\mathcal{M}_{\alpha}$ is a product of subsets $\mathcal{M}_{\alpha, i}$ of $\mathbb{N}$ which are either points or of the form $\left\{m_{i}=c_{\alpha, i} q_{\alpha, i}^{n_{i}}: n_{i} \in \mathbb{N}\right\}$ for some positive integers $c_{\alpha, i}, q_{\alpha, i}$,

- on each piece $\mathcal{M}_{\alpha}$, the volume $\omega_{m}$ is given by a formula $\prod_{i \in I_{f}} P_{\alpha, i}\left(n_{i}, q_{\alpha, i}^{n_{i} / d}\right)$ where $P_{\alpha, i}$ is a polynomial and $d$ a positive integer.

According to Lemma 7.6 with $T=2^{t}$, the volume

$$
w_{\alpha, T}:=\sum_{\left\{m \in \mathcal{M}_{\alpha}, \pi_{m} \leq T\right\}} \omega_{m}
$$

satisfy the bound $w_{\alpha, 2 T}=O\left(w_{\alpha, T}\right)$. Hence one has $w_{2 T}=O\left(w_{T}\right)$ as required.

(4): Let $T_{0}:=\inf _{z \in Z_{\infty}} h_{\infty}(z)>0$. According to (2), there exists $C>0$ such that for $T$ large

$$
\begin{aligned}
V_{(1+\varepsilon) T}-V_{T} & =\sum_{m}\left(v_{(1+\varepsilon) T / \pi_{m}}-v_{T / \pi_{m}}\right) \omega_{m} \\
& \leq C \varepsilon^{\kappa}\left(\sum_{m} v_{T / \pi_{m}} \omega_{m}+\sum_{m} \omega_{m}\right) \\
& \leq C \varepsilon^{\kappa}\left(V_{T}+w_{2 T / T_{0}}\right)
\end{aligned}
$$

where the above sums are over all the multi-indices $m \in \mathbb{N}^{I_{f}}$ with $T_{0} \pi_{m} \leq 2 T$. Then, applying (3) twice, there exists $C^{\prime}>0$ such that for $T$ large

$$
\begin{aligned}
V_{(1+\varepsilon) T}-V_{T} & \leq C \varepsilon^{\kappa}\left(V_{T}+C^{\prime} w_{T / 2 T_{0}}\right) \\
& \leq C\left(1+C^{\prime} v_{2 T_{0}}^{-1}\right) \varepsilon^{\kappa} V_{T},
\end{aligned}
$$

as required, since $v_{2 T_{0}}>0$. 
Remark 7.10. One has the following estimate for the volume $V_{T}$ of the height ball : there exist $a \in \mathbb{Q}_{\geq 0}, b \in \mathbb{Z}_{\geq 0}$ and $c_{1}, c_{2}>0$ such that for $T$ large,

$$
c_{1} T^{a} \log (T)^{b} \leq V_{T} \leq c_{2} T^{a} \log (T)^{b} .
$$

This is a straightforward consequence of the formula $V_{T}:=\int_{0}^{\infty} w_{T / t} v_{t}^{\prime} d t$ and of the estimation of $w_{T}$ and $v_{T}^{\prime}$ given in Remark 7.7 and Lemma 7.8 (1).

Proposition 7.11. (Height ball) The family of height balls $B_{T}:=\{z \in Z \mid$ $h(z) \leq T\}, T \gg 1$, is well rounded.

Proof. We will assume as we may that all the orbits $Z_{i}$ have positive dimension. When $I_{\infty}=\emptyset$, the well-roundedness of $B_{T}$ is a consequence of Example 7.5. Hence we will assume that $I_{\infty} \neq \emptyset$. When the height function $h_{\infty}$ is constant on $Z_{\infty}$, the well-roundedness of $B_{T}$ is a consequence of Example 7.1 .

When the height function $h_{\infty}$ is not constant on $Z_{\infty}$, the well-roundedness of $B_{T}$ follows from Lemma 7.8 (4) and of the linearity of the action of each $G_{i}$ on $V_{i}$.

Although we stated the above proposition only for characteristic 0 fields, when all the $k_{i}$ have positive characteristic, the height balls are also well-rounded by Example 7.5.

\section{Applications}

We will be applying the following theorem and corollary to the above examples of well rounded sequences.

Theorem 8.1. Let $I$ be a finite set. For each $i \in I$, let $k_{i}$ be a local field of characteristic not $2, G_{i}$ the group of $k_{i}$-points of a semisimple algebraic $k_{i}$ group, $H_{i} \subset G_{i}$ the $k_{i}$-points of a symmetric $k_{i}$-subgroup. Set $G_{I}:=\prod_{i \in I} G_{i}$, $H_{I}:=\prod_{i \in I} H_{i}, Z_{I}:=H_{I} \backslash G_{I}$ and $z_{0}=\left[H_{I}\right]$. Let $\Gamma$ be an irreducible lattice of $G_{I}$ such that $\Gamma_{H}:=\Gamma \cap H_{I}$ is a lattice in $H_{I}$. Then for any sequence $B_{n}$ of well-rounded subsets of $Z_{I}$ with volume tending to infinity, we have, as $n \rightarrow \infty$,

$$
\#\left(z_{0} \Gamma \cap B_{n}\right) \sim \frac{\mu_{Y}(Y)}{\mu_{X}(X)} \mu_{Z_{I}}\left(B_{n}\right),
$$

where $X=\Gamma \backslash G_{I}, Y=\Gamma_{H} \backslash H_{I}$ and the volumes are computed using invariant measures as in (5.2).

Proof. Use Corollary 4.6 and Proposition 6.2 with $\varphi_{n}:=\mathbf{1}_{B_{n}}$.

In the product situation of $Z_{I}=Z_{I_{0}} \times Z_{I_{1}}$, we will be taking a well-rounded sequences of $Z_{I}$ which are products of a fixed compact subset in one factor $Z_{I_{1}}$ and a well-rounded sequence of subsets in the other factor $Z_{I_{0}}$. This will give us equidistribution results in the space $Z_{I_{1}}$ when $Z_{I_{0}}$ is non-compact. 
Corollary 8.2. Keeping the same hypothesis as in Theorem 8.1, let $I=I_{0} \sqcup I_{1}$ be a partition of $I$. Letting $B_{n}$ be a well-rounded sequence of subsets of $Z_{I_{1}}$ with volume going to infinity, consider the following discrete multisets $Z(n)$ of $Z_{I_{0}}$ :

$$
Z(n):=\left\{z \in Z_{I_{0}} \mid\left(z, z^{\prime}\right) \in z_{0} \Gamma \cap\left(Z_{I_{0}} \times B_{n}\right) \quad \text { for some } z^{\prime} \in Z_{I_{1}}\right\} .
$$

Then, as $n \rightarrow \infty$, the sets $Z(n)$ become equidistributed in $Z_{I_{0}}$ with respect to a suitably normalized invariant measure. In fact, for any $\varphi \in C_{c}\left(Z_{I_{0}}\right)$,

$$
\lim _{n \rightarrow \infty} \frac{1}{\mu_{Z_{I_{1}}}\left(B_{n}\right)} \sum_{z \in Z(n)} \varphi(z)=\frac{\mu_{Y}(Y)}{\mu_{X}(X)} \int_{Z_{I_{0}}} \varphi d \mu_{Z_{I_{0}}} .
$$

In particular, $Z(n)$ is non-empty for all large $n$.

Multiset means that the points of $Z(n)$ are counted with multiplicity according to the cardinality of the fibers of the projection $z_{0} \Gamma \cap\left(Z_{I_{0}} \times B_{n}\right) \rightarrow Z(n)$. Since $z_{0} \Gamma$ is discrete and $B_{n}$ is relatively compact, we note that these fibers are finite and that $Z(n)$ is discrete in $Z_{I_{0}}$.

Proof. It suffices to prove the claim for non-negative functions $\varphi \in C_{c}\left(Z_{I_{0}}\right)$. Define a sequence of functions $\varphi_{n}$ on $Z$ by

$$
\varphi_{n}\left(z, z^{\prime}\right):=\left(\varphi \otimes \mathbf{1}_{B_{n}}\right)\left(z, z^{\prime}\right)=\varphi(z) \mathbf{1}_{B_{n}}\left(z^{\prime}\right) \quad \text { for }\left(z, z^{\prime}\right) \in Z_{I_{0}} \times Z_{I_{1}} .
$$

By Example [7.1, this sequence $\varphi_{n}$ is well-rounded and

$$
\sum_{z \in Z(n)} \varphi(z)=\sum_{z \in z_{0} \Gamma} \varphi_{n}(z)
$$

since $Z(n)$ is a multiset.

By Corollary 4.6, we can apply Proposition 6.2 to the sequence $\varphi_{n}$ and obtain

$$
\lim _{n \rightarrow \infty} \frac{1}{\mu_{Z_{1}}\left(B_{n}\right)} \sum_{z \in Z(n)} \varphi(z)=\lim _{n \rightarrow \infty} \frac{1}{\mu_{Z_{I}}\left(B_{n}\right)} \sum_{z \in z_{0} \Gamma} \varphi_{n}(z)=\frac{\mu_{Y}(Y)}{\mu_{X}(X)} \int_{Z_{I_{0}}} \varphi d \mu_{Z_{I_{0}}} .
$$

Remark 8.3. In Propositions 5.3 and 6.2 , one can replace the hypothesis "the $L^{1}$-norm of $\varphi_{n}$ goes to infinity" by the hypothesis that "the support of $\varphi_{n}$ is non-empty and goes to infinity" i.e. for every compact $C$ of $Z,\left.\varphi_{n}\right|_{C}$ is null for all $n$ large. The proof is exactly the same. A similar remark applies to Theorem 1.1, 8.1 and Corollary 8.2, This remark is useful for the non-empty spheres in Example 7.4, since it avoids to check that their volume goes to infinity with the radius.

Proof of Theorems 1.1, 1.4, 1.8 and Corollary 1.2. We are now ready to prove the non-effective statements in the introduction.

Theorem 1.1 is a consequence of Theorem 8.1 with $I=S, G_{S}=\mathbf{G}_{S}, H_{S}=\mathbf{H}_{S}$ and $\Gamma=\Gamma_{S}$. The only thing we have to check is that $\Gamma_{S}$ is an irreducible lattice in $\mathbf{G}_{S}$. This is the following classical lemma 8.4 . 
Theorem 1.4 is a consequence of Corollary 8.2 with $I=S$ and $I_{0}=S_{0}$. Note that the projection $z_{0} \Gamma \rightarrow Z_{I_{0}}$ is injective and hence the multiset $Z(n)$ is a set.

Theorem 1.8 is an immediate consequence of Corollary 4.6.

Corollary 1.2 is a consequence of Theorem 1.1 and Proposition 7.11 .

Let $\mathbf{G}$ be a connected semisimple group defined over a global field $K$, and let $S$ be a finite set of places of $K$ containing all archimedean places $v$ such that $\mathbf{G}_{v}$ is non-compact. Recall that these conditions assure that the subgroup $\mathbf{G}_{\mathcal{O}_{S}}$ is a lattice in $\mathbf{G}_{S}:=\prod_{v \in S} \mathbf{G}_{v}$.

Lemma 8.4. Let $\Gamma_{S}$ be a subgroup of finite index in $\mathbf{G}_{\mathcal{O}_{S}}$. Suppose that $\mathbf{G}$ is simply connected, almost $K$-simple and that $\mathbf{G}_{S}$ is non-compact. Then $\Gamma_{S}$ is an irreducible lattice in $\mathbf{G}_{S}$ (see Definition 2.3).

Proof. Since $\mathbf{G}$ is simply connected and $\mathbf{G}_{S}$ is non-compact, then $\mathbf{G}$ has the strong approximation property with respect to $S$, that is, the diagonal embedding of $\mathbf{G}_{K}$ is dense in the $S$-adeles $\mathbf{G}_{\mathbb{A}_{S}}$, i.e., the adeles without $S$-component (see [42, Th. 7.12] for characteristic 0 cases and [41] for the positive characteristic case). Since $\mathbf{G}$ is $K$-simple, it follows that $\Gamma_{S}$ is an irreducible lattice in $\mathbf{G}_{S}$ [35, Cor. I.2.3.2 \& Th. II.6.8].

For the rest of this paper, we will transform the proofs explained in the above chapters into effective proofs. For that we need to control precisely all the error terms appearing in these proofs. There are mainly four error terms to control. The first three come from the mixing property, the wave front property and the approximation of $\mu_{Y}$ by a smooth function. Their control will give the equidistribution speed of the translates of $\mu_{Y}$. The last error term comes from the well roundedness of the balls $B_{T}$. We will dedicate one section to each of these terms.

\section{EFFECTIVE MIXING}

In this section, we introduce notations which will be used through the section 14 and we describe an effective version (Theorem 9.2) of the mixing property based on the uniform decay of matrix coefficients.

We let $K$ be a number field, $\mathbf{G}$ a connected simply connected almost $K$-simple group and $\mathbf{H}$ a $K$-subgroup of $\mathbf{G}$ with no non-trivial $K$-character. Let $S$ be a finite set of places of $K$ containing all the infinite places $v$ such that $\mathbf{G}_{v}$ is non-compact. We write $S_{\infty}$ and $S_{f}$ for the sets of infinite and finite places in $S$ respectively. We assume that $\mathbf{G}_{S}:=\prod_{v \in S} \mathbf{G}_{v}$ is non-compact. Let $\Gamma_{S}$ be a finite index subgroup of $\mathbf{G}\left(\mathcal{O}_{S}\right)$. Note that $\mathbf{H}_{S} \cap \Gamma_{S}$ is a lattice in $\mathbf{H}_{S}$. 
Set $X_{S}:=\Gamma_{S} \backslash \mathbf{G}_{\mathbf{S}}$ and $Y_{S}=\Gamma_{S} \cap \mathbf{H}_{S} \backslash \mathbf{H}_{S}$. Let $\mu_{X_{S}}$ and $\mu_{Y_{S}}$ denote the invariant probability measures on $X_{S}$ and $Y_{S}$ respectively. Set $Z_{S}:=\mathbf{H}_{S} \backslash \mathbf{G}_{\mathbf{S}}$. For each $v \in S$, choose an invariant measure $\mu_{Z_{v}}$ on $\mathbf{H}_{v} \backslash \mathbf{G}_{v}$ so that the invariant measure $\mu_{Z_{S}}:=\prod_{v \in S} \mu_{Z_{v}}$ on $Z_{S}$ satisfies $\mu_{X_{S}}=\mu_{Y_{S}} \mu_{Z_{S}}$ locally. For $S_{0} \subset S$, we set $\mu_{Z_{S_{0}}}:=\prod_{v \in S_{0}} \mu_{Z_{v}}$.

By a smooth function on $X_{S}$ we mean a function which is smooth on each $\mathbf{G}_{\infty}$-orbit and which is invariant under a compact open subgroup of $\mathbf{G}_{f}$. The notation $C_{c}^{\infty}\left(\Gamma_{S} \backslash \mathbf{G}_{S}\right)$ denotes the set of smooth functions with compact support on $\mathbf{G}_{S}$.

For each $v \in S$, recall the "Cartan" decomposition due to Bruhat and Tits in [5] and [6]: one has $\mathbf{G}_{v}=M_{v} \Omega_{v} B_{v}^{+} M_{v}$ where $M_{v}$ is a good maximal compact subgroup, $B_{v}^{+}$a positive Weyl chamber of a maximal $K_{v}$-split torus and $\Omega_{v}$ is a finite subset in the centralizer of $B_{v}$.

For simplicity, we set $\mathbf{G}_{\infty}=\mathbf{G}_{S_{\infty}}$ and $\mathbf{G}_{f}=\mathbf{G}_{S_{f}}$. We also set $M_{\infty}:=$ $\prod_{v \in S_{\infty}} M_{v}$ and $M_{f}:=\prod_{v \in S_{f}} M_{v}$.

Let $X_{1}, \cdots, X_{d}$ be an orthonormal basis of the Lie algebra of $M_{\infty}$ with respect to an Ad-invariant scalar product. We denote by $\mathcal{D}$ the elliptic operator $\mathcal{D}:=$ $1-\sum_{i=1}^{d} X_{i}^{2}$.

Fix any closed embedding of $\mathbf{Z}=\mathbf{H} \backslash \mathbf{G}$ into a finite dimensional vector space V defined over $K$; such an embedding always exists by the well known theorem of Chevalley. To measure how far an element $z \in \mathbf{Z}_{S}$ is from the base point $z_{0}=\left[\mathbf{H}_{S}\right]$ in $Z_{S}:=\mathbf{H}_{S} \backslash \mathbf{G}_{S}$, we may use a height function

$$
\mathrm{H}_{S}(z):=\prod_{v \in S}\left\|z_{v}\right\|_{v}
$$

where $\|\cdot\|_{v}$ is a norm on $\mathbf{V}_{v}$. This norm is assumed to be euclidean when $v$ is an infinite place and a max norm when $v$ is a finite place. Note that the height function $\mathrm{H}_{S}: Z_{S} \rightarrow \mathbb{R}^{+}$is a proper function.

Theorem 9.2. There exists $\kappa>0$ and $m \in \mathbb{N}$ such that for any open compact subgroup $U_{f}$ of $\mathbf{G}_{f}$, there exists $C_{U_{f}}>0$ satisfying that for any $\psi_{1}, \psi_{2} \in$ $C_{c}^{\infty}\left(X_{S}\right)^{U_{f}}$ and any $g \in \mathbf{G}_{S}$,

$\left|\left\langle g \psi_{1}, \psi_{2}\right\rangle-\int_{X_{S}} \psi_{1} d \mu_{X_{S}} \int_{X_{S}} \psi_{2} d \mu_{X_{S}}\right| \leq C_{U_{f}} \mathrm{H}_{S}\left(z_{0} g\right)^{-\kappa}\left\|\mathcal{D}^{m}\left(\psi_{1}\right)\right\|_{L^{2}}\left\|\mathcal{D}^{m}\left(\psi_{2}\right)\right\|_{L^{2}}$.

Proof. The above claim is a straightforward consequence of Theorem 2.20 of [22] based on the results of [7] and [39]. [22, Theorem 2.20] relies on the following hypothesis: "the only character appearing in $L^{2}\left(\Gamma_{S} \backslash \mathbf{G}_{S}\right)$ is the trivial one". This hypothesis is satisfied here since the non compactness of $\mathbf{G}_{S}$ and the simplyconnectedness of $\mathbf{G}$ imply the irreducibility of $\Gamma_{S}$ by Lemma 8.4 ,

The conclusion of [22, Theorem 2.20] is the above claim where $\mathrm{H}_{S}^{-\kappa}$ is replaced by a function $\widetilde{\xi}_{\mathbf{G}}$ which is a product over $v \in S$ of bi- $M_{v}$-invariant functions $\xi_{v}^{\prime}$ 
satisfying

$$
\xi_{v}^{\prime}(a) \leq \prod_{\alpha \in Q_{v}} \alpha(a)^{-1 / 2+\varepsilon} \quad \text { for all } a \in B_{v}^{+}
$$

where $Q_{v}$ is a maximal strongly orthogonal system of the root system of $\left(\mathbf{G}_{v}, B_{v}\right)$

We only have to check that this function $\widetilde{\xi}_{\mathbf{G}}$ is bounded by a multiple of $\mathrm{H}_{S}^{-\kappa}$. For that, denote by $\rho$ the representation of $\mathbf{G}$ into $\mathbf{G L}(\mathbf{V})$ such that the stabilizer of $z_{0} \in \mathbf{V}_{K}$ is $\mathbf{H}$ and choose a weight $\lambda$ larger on $B_{v}^{+}$than any weight of $\rho$. Then there exists a positive integer $\mathrm{k}$ such that, for all $a \in B_{v}^{+}$,

$$
\left\|z_{0} \rho(a)\right\|_{v} \leq\left\|z_{0}\right\|_{v}\|\rho(a)\|_{v} \leq\left\|z_{0}\right\|_{v}|\lambda(a)|_{v} \leq\left\|z_{0}\right\|_{v} \prod_{\alpha \in Q_{v}} \alpha(a)^{k} .
$$

Since $M_{v}$ and $\Omega_{v}$ are compact subsets, by the continuity, this implies that there exists $\kappa>0$ and $c>0$ such that

$$
\xi_{v}^{\prime}(g) \leq c\left\|z_{0} \rho(g)\right\|_{v}^{-\kappa}
$$

for all $g \in \mathbf{G}_{v}$. This implies our claim.

\section{INJECTIVE RADIUS AND THE APPROXIMATION BY SMOOTH FUNCTIONS}

The aim of this section is to get an effective upper bound on the volume of the set of points in $Y_{S}$ with small injectivity radius in $X_{S}$ and approximate the characteristic function

Fix a closed embedding $\mathbf{G} \hookrightarrow \mathbf{G L}_{N}$. We may consider each element $g$ of $\mathbf{G}_{S}$ as an $|S|$-tuples of $N \times N$ matrices $g_{v}$. We also fix a norm $\|\cdot\|_{v}$ on each of these $K_{v}$-vector spaces of matrices.

For $x \in X_{S}$, consider the projection map $p_{x}: \mathbf{G}_{S} \rightarrow X_{S}$ given by $g \mapsto x g$. The injectivity radius $r_{x}$ is defined to be

$$
r_{x}=\sup \left\{r>0:\left.p_{x}\right|_{B_{r} \times M_{f}} \text { is injective }\right\}
$$

where $B_{r}=\left\{g \in \mathbf{G}_{\infty}: \max _{v \in S_{\infty}}\left\|g_{v}-e\right\|_{v} \leq r\right\}$.

Of course, this definition makes sense only when $S_{\infty}$ is non-empty. This does not matter since, when $S_{\infty}$ is empty, $X_{S}$ is compact.

Lemma 10.1. Suppose $S_{\infty} \neq \emptyset$. For any $x \in X_{S}$, one has $r_{x}>0$.

Proof. Since $\Gamma_{S}$ does not meet $\mathbf{G}_{f}$ and $\mathbf{G}_{f}$ is normal in $\mathbf{G}_{S}$, the group $\mathbf{G}_{f}$ acts freely on $X_{S}$. Hence $\left.p_{z}\right|_{\{e\} \times M_{f}}$ is injective. Since $M_{f}$ is compact and $p_{x}$ is locally injective, $\left.p_{x}\right|_{B_{r} \times M_{f}}$ is still injective for some small $r>0$.

Moreover we have a quantitative version of the above lemma.

Lemma 10.2. Suppose $S_{\infty} \neq \emptyset$. There exist $c_{1}>0, p_{1}>0$ such that for all sufficiently small $\varepsilon>0, \mu_{Y_{S}}\left(\left\{y \in Y_{S} \mid r_{y}<\varepsilon\right\}\right) \leq c_{1} \varepsilon^{p_{1}}$. 
Proof. We use the reduction theory for $\mathbf{H}_{S}$ (cf. [42]). We first recall what a Siegel set is. Let $\mathbf{A}$ be a maximal $K$-split torus of $\mathbf{H}$ and $\mathbf{P}$ a minimal parabolic subgroup containing $\mathbf{A}$. Then $\mathbf{P}=\mathbf{N R A}$ where $\mathbf{R}$ is a $\mathbb{Q}$-anisotropic reductive subgroup and $\mathbf{N}$ the unipotent radical of $\mathbf{P}$. Set $\mathbf{A}_{\infty}:=\prod_{v \in S_{\infty}} \mathbf{A}\left(K_{v}\right)$ and similarly $\mathbf{N}_{\infty}$ and $\mathbf{R}_{\infty}$. Denoting by $\Delta$ the system of simple roots of $\mathbf{H}_{\infty}$ determined by the choice of $\mathbf{P}$, we set for $t>0$,

$$
A_{t}=\left\{a \in \mathbf{A}_{\infty}: \alpha(a) \geq t \text { for all } \alpha \in \Delta\right\} .
$$

Then for a compact subset $\omega \subset \mathbf{N}_{\infty} \mathbf{R}_{\infty}$ and a maximal compact subgroup $K_{0}$ of $\mathbf{H}_{S}$, the set $\Sigma_{t}:=\omega A_{t} K_{0}$ is called a Siegel set. Now the reduction theory says that there exist $h_{1}, \cdots, h_{r} \in \mathbf{H}_{S}$, and a Siegel set $\Sigma_{t_{0}}=\omega A_{t_{0}} K_{0}$ such that

$$
\mathbf{H}_{S}=\cup_{i=1}^{r}\left(\mathbf{H}_{S} \cap \Gamma_{S}\right) h_{i} \Sigma_{t_{0}} .
$$

Let $M_{f}^{\prime}:=\cup_{i=1}^{r} h_{i} M_{f} h_{i}^{-1}$. As in Lemma 10.1, there exists $\varepsilon_{0}>0$ such that

$$
\Gamma_{S} \cap B_{\varepsilon_{0}} M_{f}^{\prime}=\{e\} .
$$

Set

$$
C_{\varepsilon}:=\cup_{i=1}^{r} h_{i}\left\{w a k \in \Sigma_{0}: t_{0} \leq \alpha(a) \leq \varepsilon^{-r_{0}} \text { for each } \alpha \in \Delta\right\},
$$

where $r_{0}>0$ is chosen independent of $\varepsilon$, so that, for all $g$ in $C_{\varepsilon}$ and $v \in S_{\infty}$, one has

$$
\left\|g_{v}\right\|_{v} \leq \varepsilon^{-1 / 4} \text { and }\left\|g_{v}^{-1}\right\|_{v} \leq \varepsilon^{-1 / 4}
$$

Let $Y_{\varepsilon}^{\prime}$ denote the image of $C_{\varepsilon}$ in $Y_{S}$ under the projection $\mathbf{H}_{S} \rightarrow Y_{S}$. The integration formula [42, p. 213] shows that for some constant $c_{1}>0$ and $p_{1}>0$,

$$
\mu_{Y_{S}}\left(Y_{S}-Y_{\varepsilon}^{\prime}\right) \leq c_{1} \varepsilon^{p_{1}} .
$$

Hence it is enough to show that for all $z \in Y_{\varepsilon}^{\prime}$, one has $r_{z} \geq \varepsilon$. Suppose $p_{z}(x)=$ $p_{z}(y)$ with $x, y \in B_{\varepsilon} M_{f}$ and write $z=\Gamma_{S} g$ for some $g \in C_{\varepsilon}$. We want to prove that $x=y$.

The element $\gamma:=g x y^{-1} g^{-1}$ belongs to $\Gamma_{S}$. Moreover, for some fixed constant $c>1$, one has, for all $v \in S_{\infty}$,

$$
\begin{aligned}
\|\gamma-e\|_{v} & =\left\|g_{v}\left(x_{v}-y_{v}\right) y_{v}^{-1} g_{v}^{-1}\right\|_{v} \\
& \leq c \varepsilon^{-1 / 2}\left\|x_{v}-y_{v}\right\|_{v}\left\|y_{v}^{-1}\right\|_{v} \\
& \leq c^{2} \varepsilon^{1 / 2}
\end{aligned}
$$

But the finite component of $\gamma$ is in $M_{f}^{\prime}$, hence, $\gamma$ is in $B_{c^{2} \varepsilon^{1 / 2}} \times M_{f}$ and one gets from (10.3) that, for $\varepsilon<c^{-4} \varepsilon_{0}^{2}$, one has $\gamma=e$. Therefore $x=y$ as well.

For all $v \in S$, we choose a small neighborhood $\mathfrak{s}_{v}$ of 0 in a supplementary subspace of the Lie algebra $\mathfrak{h}_{v}$ in $\mathfrak{g}_{v}$ and set $\mathfrak{s}:=\prod_{v \in S} \mathfrak{s}_{v}$. The set $W:=\exp (\mathfrak{s})$ is then a transversal to $\mathbf{H}_{S}$ in $\mathbf{G}_{S}$. We set $\mu_{W}$ the measure on $W$ such that $d \mu_{X_{S}}=d \mu_{Y_{S}} d \mu_{W}$ locally. 
Recall that $B_{\varepsilon}$ denotes the ball of center $e$ and radius $\varepsilon$ in $\mathbf{G}_{\infty}$ and let $U_{\varepsilon}$ be the ball of center $e$ and radius $\varepsilon$ in $\mathbf{G}_{f}$ :

$$
\begin{aligned}
& B_{\varepsilon}=\left\{g \in \mathbf{G}_{\infty} \mid \max _{s \in S_{\infty}}\|g-e\|_{v} \leq \varepsilon\right\}, \\
& U_{\varepsilon}=\left\{g \in \mathbf{G}_{f} \mid \max _{s \in S_{f}}\|g-e\|_{v} \leq \varepsilon\right\} .
\end{aligned}
$$

We fix $\varepsilon_{0}>0$ small. For $\varepsilon$ small we let

$$
H_{\varepsilon}:=\mathbf{H}_{S} \cap B_{\varepsilon} U_{\varepsilon_{0}} \quad \text { and } \quad W_{\varepsilon}:=W \cap B_{\varepsilon} U_{\varepsilon_{0}}
$$

so that the multiplication $H_{\varepsilon} \times W_{\varepsilon} \rightarrow H_{\varepsilon} W_{\varepsilon}$ is an homeomorphism onto a neighborhood of $e$. Fix $m>\operatorname{dim} \mathbf{G}_{\infty}$ and $\kappa>0$ satisfying Theorem 9.2 and fix $l \in \mathbb{N}$ as in Lemma 11.3. We can assume that $U_{l \varepsilon_{0}} \subset U_{f} \cap M_{f}$ and let $U_{f}^{\prime}$ be an open subgroup of $\mathbf{G}_{f}$ such that $U_{\varepsilon_{0}} U_{f}^{\prime}=U_{\varepsilon_{0}}$.

Lemma 10.6. Let $Y_{\varepsilon}:=\left\{y \in Y_{S} \mid\right.$ the map $g \mapsto y g$ is injective on $\left.H_{\varepsilon} W_{\varepsilon}\right\}$. There exist $c_{1}>0$ and $p_{1}>0$ such that for all small $\varepsilon>0$,

$$
\mu_{Y_{S}}\left(Y_{S}-Y_{\varepsilon}\right) \leq c_{1} \varepsilon^{p_{1}} .
$$

Proof. When $S_{\infty} \neq \emptyset$, since $B_{\varepsilon} B_{\varepsilon} \subset B_{\varepsilon^{1 / 2}}$ for $\varepsilon$ small, the set $Y_{\varepsilon}$ contains the set of points $y$ such that $r_{y} \geq \varepsilon^{1 / 2}$. Just apply then Lemma 10.2.

When $S_{\infty}=\emptyset, X_{S}$ is compact, hence $Y_{\varepsilon}$ is equal to $Y_{S}$ for $\varepsilon_{0}$ and $\varepsilon$ small.

The following proposition provides the approximation of the characteristic function $\mathbf{1}_{Y_{\varepsilon} W}$ by a smooth function $\varphi_{\varepsilon}$ with the controlled Sobolev norm.

We first recall the Sobolev norm $\mathcal{S}_{m}(\psi)$ of a function $\psi \in C_{c}^{\infty}\left(X_{S}\right)$. Choose a basis $X_{1}, \ldots, X_{n}$ of the Lie algebra of $\mathbf{G}_{\infty}$. For each $k$-tuple of integers $a:=$ $\left(a_{1}, \ldots, a_{k}\right)$ with $1 \leq a_{i} \leq n$, the product $X_{a}:=X_{a_{1}} \ldots X_{a_{k}}$ defines a left-invariant differential operator on $\mathbf{G}_{S}$, hence a differential operator on $X_{S}$. By definition $\mathcal{S}_{m}(\psi)^{2}=\sum_{a}\left\|X_{a} \psi\right\|_{L^{2}}^{2}$ where the sum is over all the $k$-tuples $a$ with $0 \leq k \leq m$ and where $X_{\emptyset} \psi$ stands for $\psi$.

Proposition 10.7. There exist $p_{2}>0$ such that, for all sufficiently small $\varepsilon$, one can choose

- a non-negative smooth function $\rho_{\varepsilon}$ on $W$ with support in $W_{\varepsilon}$ such that $\int_{W} \rho_{\varepsilon}=1$, - a non-negative smooth function $\tau_{\varepsilon}$ on $Y_{S}$ with support in $Y_{\varepsilon}$ such that $\tau_{\varepsilon} \leq 1$ on $Y_{S}$ and $\left.\tau_{\varepsilon}\right|_{Y_{4 \varepsilon}}=1$.

- Moreover, let $\varphi_{\varepsilon}$ be the function on $X_{S}$ defined by

$$
\varphi_{\varepsilon}(x)=\sum_{\left\{(y, w) \in Y_{\varepsilon} \times W_{\varepsilon} \mid y w=x\right\}} \tau_{\varepsilon}(y) \rho_{\varepsilon}(w) .
$$

The choices can be made so that $\varphi_{\varepsilon}$ is $U_{f}^{\prime}$-invariant and $\mathcal{S}_{m}\left(\varphi_{\varepsilon}\right) \leq \varepsilon^{-p_{2}}$.

We remark that the sum defining $\varphi_{\varepsilon}$ is a finite sum and hence $\varphi_{\varepsilon}$ is well defined.

To prove Proposition 10.7, we first need a lemma which constructs some test functions $\alpha_{\varepsilon}$ near $e$. 
Lemma 10.8. For a given $m \geq 0$, there exists $p \in \mathbb{N}$, such that, for all sufficiently small $\varepsilon>0$, one can choose smooth non-negative functions $\beta_{\varepsilon}$ on $H_{\varepsilon}$ and smooth non-negative functions $\rho_{\varepsilon}$ on $W_{\varepsilon}$ satisfying the following:

- one has $\beta_{\varepsilon} \geq 1$ on $H_{\varepsilon^{2}}$.

- one has $\int_{W} \rho_{\varepsilon} d \mu_{W}=1$

- if $\alpha_{\varepsilon}$ denotes the smooth function on $H_{\varepsilon} W_{\varepsilon}$ given by $\alpha_{\varepsilon}(h w)=\beta_{\varepsilon}(h) \rho_{\varepsilon}(w)$, then $\alpha_{\varepsilon}$ is $U_{f}^{\prime}$-invariant and $\mathcal{S}_{m}\left(\alpha_{\varepsilon}\right) \leq \varepsilon^{-p}$.

Proof. The general case reduces to the case of $S=S_{\infty}$, by considering tensor products with characteristic functions of $U_{\varepsilon_{0}} \cap H$ and of $U_{\varepsilon_{0}} \cap W$. Hence, we can assume that $S=S_{\infty}$ so that $\mathbf{G}_{S}$ is a real Lie group. Set $d=\operatorname{dim} W$. Fix some smooth non-negative functions $\beta$ on $\mathfrak{h}:=\oplus_{v \in S} \mathfrak{h}_{v}$ and $\rho$ on $\mathfrak{s}$ with support in a sufficiently small neighborhood of 0 such that $\beta(0)>1$ and $\int_{\mathfrak{s}} \rho=1$. Then, for suitable constants $c_{\varepsilon}>0$ converging to 1 , the functions given by

$$
\beta_{\varepsilon}(\exp (X))=\beta(X / \varepsilon) \quad \text { and } \quad \rho_{\varepsilon}(\exp (Y))=c_{\varepsilon} \varepsilon^{-d} \rho(Y / \varepsilon),
$$

for $X$ (resp. $Y$ ) in a fixed compact neighborhood of 0 in $\mathfrak{h}$ (resp. $\mathfrak{s}$ ), satisfy the properties listed above.

Proof of Proposition 10.7. We choose the function $\rho_{\varepsilon}$ from Lemma 10.8. To construct the function $\tau_{\varepsilon}$, consider a maximal family $\mathcal{G}_{\varepsilon}$ of points $y \in Y_{\varepsilon}$ such that the subsets $y H_{\varepsilon^{3}}$ of $Y_{S}$ are disjoint and meet $Y_{2 \varepsilon}$ and let $\mathcal{F}_{\varepsilon} \subset \mathcal{G}_{\varepsilon}$ the subfamily for which $y H_{\varepsilon^{3}}$ meets $Y_{4 \varepsilon}$. For all $y \in \mathcal{G}_{\varepsilon}$ the volumes $\mu_{Y_{S}}\left(y H_{\varepsilon^{3}}\right)$ are equal and of order $\varepsilon^{3 d}$ with $d=\operatorname{dim}\left(\mathbf{H}_{\infty}\right)$. Since $\mu_{Y_{S}}\left(Y_{S}\right)=1$, the cardinality of $\mathcal{G}_{\varepsilon}$ is at most $O\left(\varepsilon^{-3 d}\right)$.

For $y \in \mathcal{G}_{\varepsilon}$ we define a test function $\beta_{y, \varepsilon}$ on $Y_{S}$ with support on $y H_{\varepsilon}$ by $\beta_{y, \varepsilon}(y h)=\beta_{\varepsilon}(h)$ and let $\beta_{\mathcal{G}, \varepsilon}:=\sum_{y \in \mathcal{G}_{\varepsilon}} \beta_{y, \varepsilon}$. Since $B_{\varepsilon^{3}} B_{\varepsilon^{3}} \subset B_{\varepsilon^{2}}$, the sets $y H_{\varepsilon^{2}}$, $y \in \mathcal{G}_{\varepsilon}$, cover $Y_{2 \varepsilon}$. Hence $\beta_{\mathcal{G}, \varepsilon} \geq 1$ on $Y_{2 \varepsilon}$.

For each $y \in \mathcal{F}_{\varepsilon}$, consider the test function $\tau_{y, \varepsilon}$ on $Y_{S}$ with support in $Y_{2 \varepsilon}$ given by $\tau_{y, \varepsilon}:=\beta_{y, \varepsilon} / \beta_{\mathcal{G}, \varepsilon}$ on $Y_{2 \varepsilon}$ and set

$$
\tau_{\varepsilon}:=\sum_{y \in \mathcal{F}_{\varepsilon}} \tau_{y, \varepsilon}
$$

Note that $0 \leq \tau_{\varepsilon} \leq 1$ on $Y_{S},\left.\tau_{\varepsilon}\right|_{Y_{4} \varepsilon}=1$ and $\left.\tau_{\varepsilon}\right|_{Y_{S}-Y_{\varepsilon}}=0$. For $y \in \mathcal{F}_{\varepsilon}$, we also define the test function $\varphi_{y, \varepsilon}$ on $X_{S}$ with support on $y H_{\varepsilon} W_{\varepsilon}$ given by

$$
\varphi_{y, \varepsilon}(y h w):=\tau_{y, \varepsilon}(y h) \rho_{\varepsilon}(w)=\alpha_{\varepsilon}(h w) / \beta_{\mathcal{G}, \varepsilon}(y h) .
$$

These functions $\varphi_{y, \varepsilon}$ are well-defined since $y$ belongs to the set $Y_{\varepsilon}$ given by Lemma 10.6. By construction, we have

$$
\varphi_{\varepsilon}=\sum_{y \in \mathcal{F}_{\varepsilon}} \varphi_{y, \varepsilon} .
$$

It follows from $\mathcal{S}_{m}\left(\alpha_{\varepsilon}\right) \leq \varepsilon^{-p}$ that there exists $p_{0}>0$ such that

$$
\max _{y \in \mathcal{F}_{\varepsilon}} \mathcal{S}_{m}\left(\varphi_{y, \varepsilon}\right)=O\left(\varepsilon^{-p_{0}}\right)
$$


and hence $\mathcal{S}_{m}\left(\varphi_{\varepsilon}\right)=O\left(\varepsilon^{-3 d-p_{0}}\right)$.

\section{Effective equidistribution of translates of $\mathbf{H}_{S}$-Orbits}

The goal of this section is to prove Theorem 1.9, or its stronger version Theorem 11.5 below. This is an effective version of Proposition 4.1 on the equidistribution of translates of $\mathbf{H}_{S^{-}}$orbits in $X_{S}$.

Definition 11.1. We say that the translates $Y_{S} g$ are effectively equidistributed in $X_{S}$ as $g \rightarrow \infty$ in $Z_{S}$ if there exists $m \in \mathbb{N}$ and $r>0$ such that, for any compact open subgroup $U_{f}$ of $\mathbf{G}_{f}$ and any compact subset $C$ of $X_{S}$, there exists $c=c\left(U_{f}, C\right)>0$ satisfying that for any smooth function $\psi \in C_{c}^{\infty}\left(X_{S}\right)^{U_{f}}$ with support in $C$, one has for all $g \in \mathbf{G}_{S}$

$$
\left|\int_{Y_{S}} \psi(y g) d \mu_{Y_{S}}(y)-\int_{X_{S}} \psi d \mu_{X_{S}}\right| \leq c S_{m}(\psi) \mathrm{H}_{S}\left(z_{0} g\right)^{-r} .
$$

Assume further that $\mathbf{H}$ is a symmetric $K$-subgroup of $\mathbf{G}$. Taking the product of the polar decompositions $\mathbf{G}_{v}=\mathbf{H}_{v} A_{v} K_{v}$ given in Lemma 3.3 over $v \in S$, we obtain a polar decomposition of the shape

$$
\mathbf{G}_{S}=\mathbf{H}_{S} A_{S} K_{S} \text { and we set } F_{S}:=A_{S} K_{S} .
$$

The following effective version of the wavefront property 3.1 is a main technical reason why our proof of Theorem 11.5 works for $\mathbf{H}$ a symmetric subgroup.

Lemma 11.3. There exists $l \in \mathbb{N}$ such that for all small $\varepsilon, \varepsilon^{\prime}>0$ and all $g \in F_{S}$,

$$
\mathbf{H}_{S} B_{\varepsilon / l} U_{\varepsilon^{\prime} / l} g \subset \mathbf{H}_{S} g B_{\varepsilon} U_{\varepsilon^{\prime}} .
$$

Proof. We only have to check this separately at each place $v$. This statement is then a strengthening of Proposition 3.2 on the wavefront property and is an output of the proof of this Proposition.

Theorem 11.5. If $\mathbf{H}$ is a symmetric $K$-subgroup of $\mathbf{G}$, then the translates $Y_{S} g$ are effectively equidistributed in $X_{S}$ as $g \rightarrow \infty$ in $Z_{S}$.

Proof. Since $\mathbf{G}_{S}=\mathbf{H}_{S} F_{S}$, it suffices to prove the above claim for $g \in F_{S}$. We may also assume that $\int_{X_{S}} \psi d \mu_{X_{S}}=1$. We want to bound $\left|I_{g}-1\right|$ where

$$
I_{g}:=\int_{Y_{S}} \psi(y g) d \mu_{Y_{S}}(y) .
$$

We follow the proof of Proposition 4.1. The main modification will be to replace the characteristic function $\mathbf{1}_{Y_{\varepsilon} W}$ by the test function $\varphi_{\varepsilon}$ constructed in Proposition 10.7. By the same argument as in section 4, but using the stronger version 11.3 of the wavefront lemma, we have that for all small $\varepsilon>0$ and for any $w \in W_{\varepsilon}$

$$
\left|I_{g}-\int_{Y_{S}} \psi(y w g) d \mu_{Y_{S}}(y)\right| \leq l \varepsilon C_{\psi} .
$$


Here $C_{\psi}$ is the Lipschitz constant at $\infty$, i.e. the smallest constant such that for all $\varepsilon>0,|\psi(x u)-\psi(x)| \leq C_{\psi} \varepsilon$ for all $x \in X_{S}$ and $u \in B_{\varepsilon}$.

Set $\tau_{\varepsilon}, \rho_{\varepsilon}$ and $\varphi_{\varepsilon}$ the functions constructed in Proposition 10.7 and

$$
J_{g, \varepsilon}:=\int_{W_{\varepsilon}} \int_{Y_{S}} \psi(y w g) \rho_{\varepsilon}(w) d \mu_{Y_{S}}(y) d \mu_{W}(w) .
$$

By integrating (11.6) against $\rho_{\varepsilon}$, we obtain

$$
\left|I_{g}-J_{g, \varepsilon}\right| \leq l \varepsilon C_{\psi}
$$

Set also

$$
\begin{aligned}
K_{g, \varepsilon} & :=\int_{X_{S}} \psi(x g) \varphi_{\varepsilon}(x) d \mu_{X_{S}}(x) \\
& =\int_{W_{\varepsilon}} \int_{Y_{S}} \psi(y w g) \tau_{\varepsilon}(y) \rho_{\varepsilon}(w) d \mu_{Y_{S}}(y) d \mu_{W}(w) .
\end{aligned}
$$

Noting that $\tau_{\varepsilon}(y)=1$ for $y \in Y_{4 \varepsilon}$, we have for some $c_{1}, p_{1}>0$

$$
\begin{aligned}
\left|J_{g, \varepsilon}-K_{g, \varepsilon}\right| & =\left|\int_{W_{\varepsilon}} \int_{Y_{S}} \psi(y w g)\left(1-\tau_{\varepsilon}(y)\right) \rho_{\varepsilon}(w) d \mu_{Y_{S}}(y) d \mu_{W}(w)\right| \\
& \leq 2 \mu_{Y_{S}}\left(Y_{S}-Y_{4 \varepsilon}\right)\left(\int_{W_{\varepsilon}} \rho_{\varepsilon}\right)\|\psi\|_{\infty} \\
& \leq c_{1} \varepsilon^{p_{1}}\|\psi\|_{\infty} .
\end{aligned}
$$

Note that $K_{g, \varepsilon}=\left\langle g . \psi, \varphi_{\varepsilon}\right\rangle$. Since $\varphi_{\varepsilon}$ and $\psi$ are $U_{f}^{\prime}$-invariant, by Theorem 9.2 and Proposition 10.7, we deduce for some $c^{\prime}, c_{2}, p_{2}>0$

$$
\begin{aligned}
\left|K_{g, \varepsilon}-\int_{X_{S}} \varphi_{\varepsilon} d \mu_{X_{S}}\right| & \leq c^{\prime}\left\|\mathcal{D}^{m}(\psi)\right\|_{L^{2}}\left\|\mathcal{D}^{m}\left(\varphi_{\varepsilon}\right)\right\|_{L^{2}} \mathrm{H}_{S}\left(z_{0} g\right)^{-\kappa} \\
& \leq c_{2} \varepsilon^{-p_{2}}\left\|\mathcal{D}^{m}(\psi)\right\|_{L^{2}} \mathrm{H}_{S}\left(z_{0} g\right)^{-\kappa}
\end{aligned}
$$

Moreover, one has

$$
\left|\int_{X_{S}} \varphi_{\varepsilon} d \mu_{X_{S}}-1\right|=\left|\int_{Y_{S}} \tau_{\varepsilon}(y) d \mu_{Y_{S}}(y)-1\right| \leq \mu_{Y_{S}}\left(Y-Y_{4 \varepsilon}\right) \leq c_{1} \varepsilon^{p_{1}} .
$$

Since $C$ is compact, the $C^{1}$-norm of a $U_{f}^{\prime}$-invariant function $\psi$ supported on $C$ is bounded above by a uniform multiple of a suitable Sobolev norm as in [2, Theorem 2.20] i.e., one has an inequality

$$
\max \left(\|\psi\|_{\infty}, C_{\psi}\right) \leq c^{\prime \prime} \mathcal{S}_{2 m}(\psi)
$$


with $c^{\prime \prime}=c^{\prime \prime}\left(U_{f}, C\right)>0$ independent of $\psi$. Hence, putting all these upper bounds together and using the inequality $1 \leq\|\psi\|_{\infty}$, we get

$$
\begin{aligned}
\left|I_{g}-1\right| & \leq\left|I_{g}-J_{g, \varepsilon}\right|+\left|J_{g, \varepsilon}-K_{g, \varepsilon}\right|+\left|K_{g, \varepsilon}-\int \varphi_{\varepsilon}\right|+\left|\int \varphi_{\varepsilon}-1\right| \\
& \leq l \varepsilon C_{\psi}+c_{1} \varepsilon^{p_{1}}\|\psi\|_{\infty}+c_{2} \varepsilon^{-p_{2}}\left\|\mathcal{D}^{m}(\psi)\right\|_{L^{2}} \mathrm{H}_{S}\left(z_{0} g\right)^{-\kappa}+c_{1} \varepsilon^{p_{1}} \\
& \leq\left(c_{1}^{\prime} \varepsilon^{p_{1}}+c_{2}^{\prime} \varepsilon^{-p_{2}} \mathrm{H}_{S}\left(z_{0} g\right)^{-\kappa}\right) \mathcal{S}_{2 m}(\psi) .
\end{aligned}
$$

Note in the above that the positive constants $c_{i}^{\prime}, p_{i}, i=1,2$, are independent of $\psi$.

Now by taking $\varepsilon=\mathrm{H}_{S}\left(z_{0} g\right)^{-r / p_{1}}$ with $r=\frac{\kappa p_{1}}{p_{1}+p_{2}}$, we obtain as required

$$
\left|I_{g}-1\right| \leq c \mathcal{S}_{2 m}(\psi) \mathrm{H}_{S}\left(z_{0} g\right)^{-r} .
$$

This concludes the proof.

\section{Remarks}

(1) One could also, as an output of our proof, compute explicitly $m$ and $r$ and describe how the constant $c$ depends on the compact sets $U_{f}$ and $C$.

(2) Note that the above theorem [11.5 is precisely the effective version of Proposition 4.1. since we have shown that the effective mixing theorem 9.2 together with the effective wave front lemma 11.3 imply the effective equidistribution of $Y_{S} g$.

\section{EfFECTIVE COUNTING AND EQUIDISTRIBUtion}

The following definition is an effective version of Definition 6.1. Recall that $B_{\varepsilon}=B(e, \varepsilon)$ is the ball of center $e$ and radius $\varepsilon$ in $\mathbf{G}_{\infty}$ (10.4) and that $\mathrm{H}_{S}$ is a height function on $Z_{S}$ as defined in (9.1).

Definition 12.1. A sequence of subsets $B_{n}$ in $Z_{S}$ is said to be effectively wellrounded if

(1) it is invariant under a compact open subgroup of $\mathbf{G}_{f}$,

(2) there exists $\kappa>0$ such that, uniformly for all $n \geq 1$ and all $\varepsilon \in] 0,1[$,

$$
\mu_{Z_{S}}\left(B_{n, \varepsilon}^{+}-B_{n, \varepsilon}^{-}\right)=O\left(\varepsilon^{\kappa} \mu_{Z_{S}}\left(B_{n}\right)\right)
$$

where $B_{n, \varepsilon}^{+}=B_{n} B_{\varepsilon}$ and $B_{n, \varepsilon}^{-}=\cap_{u \in B_{\varepsilon}} B_{n} u$,

(3) for any $k>0$, there exists $\delta>0$ such that, uniformly for all $n \gg 1$ and all $\varepsilon \in] 0,1[$, one has

$$
\int_{B_{n, \varepsilon}^{+}} \mathrm{H}_{S}^{-k}(z) d \mu_{Z_{S}}(z)=O\left(\mu_{Z_{S}}\left(B_{n}\right)^{1-\delta}\right) .
$$

If $S_{\infty}$ is empty, then the assumption (2) is void.

A subset $\Omega$ of $Z_{S}$ is said to be effectively well-rounded if the constant sequence $B_{n}=\Omega$ is effectively well-rounded. This means that $\Omega$ is of non-empty interior 
and that the volume $\mu_{Z_{S}}\left(\partial_{\varepsilon} \Omega\right)$ of the $\varepsilon$-neighborhood of the boundary of $\Omega$ is a $O\left(\varepsilon^{\kappa}\right)$ for $\varepsilon$ small. For instance, a compact subset of $Z_{S_{\infty}}$ with piecewise smooth (or even piecewise $\mathrm{C}^{1}$ ) boundary is effectively well-rounded in $Z_{S_{\infty}}$.

Theorem 12.2. Suppose that the translates $Y_{S} g$ become effectively equidistributed in $X_{S}$ as $g \rightarrow \infty$ in $Z_{S}$. Then for any effectively well-rounded sequence of subsets $B_{n}$ in $Z_{S}$ such that $\operatorname{vol}\left(B_{n}\right) \rightarrow \infty$ there exists a constant $\delta_{0}>0$ such that

$$
\# z_{0} \Gamma_{S} \cap B_{n}=\operatorname{vol}\left(B_{n}\right)\left(1+O\left(\operatorname{vol}\left(B_{n}\right)^{-\delta_{0}}\right)\right) .
$$

Proof. Set $\Gamma_{S, H}:=\Gamma_{S} \cap \mathbf{H}_{S}$. As in sections 5 and 6, we define a function $F_{n}$ on $X_{S}=\Gamma_{S} \backslash \mathbf{G}_{S}$ by

$$
F_{n}\left(x_{0} g\right)=\sum_{\gamma \in \Gamma_{S, H} \backslash \Gamma_{S}} \mathbf{1}_{B_{n}}\left(z_{0} \gamma g\right), \text { for } g \in \mathbf{G}_{S} .
$$

For instance, one has

$$
F_{n}\left(x_{0}\right)=\# z_{0} \Gamma_{S} \cap B_{n} .
$$

Let $m$ and $r$ be the integers given by Theorem 11.5 and $U_{f}$ a compact open subgroup of $\mathbf{G}_{f}$. By Lemma 10.8, there exists $p>0$, a smooth $U_{f}$-invariant function $\alpha_{\varepsilon}$ on $\mathbf{G}_{S}$, supported on $B_{\varepsilon} U_{f}$ such that $\int_{\mathbf{G}_{S}} \alpha_{\varepsilon}=1$ and $\mathcal{S}_{m}\left(\alpha_{\varepsilon}\right) \leq \varepsilon^{-p}$. Here we take $\varepsilon$ and $U_{f}$ small enough so that $B_{\varepsilon} U_{f}$ injects to $X_{S}$, and hence we may consider $\alpha_{\varepsilon}$ as a function on $X_{S}$.

We also introduce the functions $F_{n}^{ \pm}$on $X_{S}$ :

$$
F_{n, \varepsilon}^{ \pm}\left(x_{0} g\right)=\sum_{\gamma \in \Gamma_{S, H} \backslash \Gamma_{S}} \mathbf{1}_{B_{n, \varepsilon}^{ \pm}}\left(z_{0} \gamma g\right) \quad, \text { for } g \in \mathbf{G}_{S}
$$

Then

and hence

$$
F_{n, \varepsilon}^{-}\left(x_{0} g\right) \leq F_{n}\left(x_{0}\right) \leq F_{n, \varepsilon}^{+}\left(x_{0} g\right) \quad \text { for all } g \in B_{\varepsilon} \times U_{f}
$$

Note that

$$
\left\langle F_{n, \varepsilon}^{-}, \alpha_{\varepsilon}\right\rangle \leq F_{n}\left(x_{0}\right) \leq\left\langle F_{n, \varepsilon}^{+}, \alpha_{\varepsilon}\right\rangle
$$

$$
\left\langle F_{n, \varepsilon}^{ \pm}, \alpha_{\varepsilon}\right\rangle=\int_{B_{n, \varepsilon}^{ \pm}}\left(\int_{Y_{S}} \alpha_{\varepsilon}(y g) d \mu_{Y_{S}}(y)\right) d \mu_{Z_{S}}\left(z_{0} g\right) .
$$

Set $v_{n}:=\operatorname{vol}\left(B_{n}\right)$ and $v_{n, \varepsilon}^{ \pm}:=\operatorname{vol}\left(B_{n, \varepsilon}^{ \pm}\right)$. Then by Theorem 11.5 and the assumptions (2) and (3) of the definition [12.1, there exist positive constants $\kappa, \delta$ and $c_{i}$ such that for all $n \gg 1$ and small $\varepsilon>0$,

$$
\begin{aligned}
\left|\left\langle F_{n, \varepsilon}^{ \pm}, \alpha_{\varepsilon}\right\rangle-v_{n}\right| & \leq \int_{B_{n, \varepsilon}^{+}}\left|\int_{Y_{S}}\left(\alpha_{\varepsilon}(y g)-1\right) d \mu_{Y_{S}}(y)\right| d \mu_{Z_{S}}\left(z_{0} g\right)+\left(v_{n, \varepsilon}^{+}-v_{n, \varepsilon}^{-}\right) \\
& \leq c \mathcal{S}_{m}\left(\alpha_{\varepsilon}\right) \int_{B_{n, \varepsilon}^{+}} \mathrm{H}_{S}(z)^{-k} d \mu_{Z_{S}}(z)+c_{2} \varepsilon^{\kappa} v_{n} \\
& \leq c_{1} \varepsilon^{-p} v_{n}^{1-\delta}+c_{2} \varepsilon^{\kappa} v_{n}
\end{aligned}
$$


Setting $\delta_{0}:=\frac{\delta}{1+p / \kappa}$ and choosing $\varepsilon=v_{n}^{-\delta_{0} / \kappa}$ we get $F_{n}\left(x_{0}\right)=v_{n}\left(1+O\left(v_{n}^{-\delta_{0}}\right)\right)$.

Corollary 12.3. Let $S=S_{0} \sqcup S_{1}$ be a partition of $S$. There exist $\delta_{0}, c>0$ such that for any effectively well-rounded sequence of subsets $B_{n}$ in $Z_{S_{1}}$ whose volumes $v_{n}:=\mu_{Z_{S_{1}}}\left(B_{n}\right)$ tend to $\infty$ and for any compact effectively well-rounded subset $\Omega$ of $Z_{S_{0}}$, we have

$$
\# z_{0} \Gamma_{S} \cap\left(\Omega \times B_{n}\right)=\frac{\mu_{Y_{S}}\left(Y_{S}\right)}{\mu_{X_{S}}\left(X_{S}\right)} v_{n} \mu_{Z_{S_{0}}}(\Omega)\left(1+O\left(v_{n}^{-\delta_{0}}\right)\right) .
$$

Proof. To apply Theorem 12.2, we only have to check that the sequence $A_{n}:=$ $\Omega \times B_{n}$ of subsets of $Z_{S}$ is effectively well-rounded, which is straightforward.

\section{EFFECTIVE WELL-ROUNDEDNESS}

In this section, we give explicit examples of effectively well-rounded families (see Definition 12.1).

We keep the notations for $K, S, \mathbf{G}, \mathbf{H}, Z, \mathrm{H}_{S}(z)=\prod_{v \in S}\|z\|_{v}$ etc., from the beginning of section 9 .

We also set for $T>0$,

$$
B_{v}(T):=\left\{z \in Z_{v} \mid\|z\|_{v} \leq T\right\} \quad \text { and } \quad S_{v}(T):=\left\{z \in Z_{v} \mid\|z\|_{v}=T\right\} .
$$

Proposition 13.2. (1) Fix a subset $S_{0} \subset S$ containing $S_{\infty}$. For an $|S|$-tuple $m=\left(m_{v}\right)$ of positive numbers, define

$$
Z(m):=\prod_{v \in S_{0}} B_{v}\left(m_{v}\right) \times \prod_{v \in S \backslash S_{0}} S_{v}\left(m_{v}\right) .
$$

Then the family of sets $Z(m), m_{v} \gg 1$, provided non-empty, is effectively well-rounded.

(2) The family of height balls $B_{T}=\left\{z \in Z_{S}: \mathrm{H}_{S}(z) \leq T\right\}$ is effectively well-rounded.

Proof. The proof relies heavily on the appendix 16. For (1), we may assume that $S$ contains only one place $v$. When $v$ is infinite, the condition 12.1 (2) is Lemma 7.8 (2) and 12.1 (3) is Corollary 16.3. c. When $v$ is finite, the condition [12.1 (2) is empty and the condition 12.1 (3) is Corollary 16.7,b.

For (2), 12.1 (2) is Lemma 7.8 (4), and 12.1 (3) is a combination of the following lemma 13.3 with the facts that, on one hand one has $B_{T, \varepsilon} \subset B_{k T}$ for some fixed $k>0$ and, on the other hand, one has $V_{k T}=O\left(V_{T}\right)$ again by Lemma 7.8 (4).

Lemma 13.3. Let $B_{T}=\left\{z \in Z_{S}: H_{S}(z) \leq T\right\}$ and $V_{T}:=\mu_{Z_{S}}\left(B_{T}\right)$. Then, for any $k>0$, there exists $\delta>0$ such that

$$
\int_{B_{T}} \mathrm{H}_{S}(z)^{-k} d \mu_{Z_{S}}(z)=O\left(V_{T}^{1-\delta}\right)
$$


Proof. We may assume that, for all $v$ in $S$ and $z$ in $Z_{v}$, one has $\|z\|_{v} \geq 1$. Set $b_{T}=\left\{z \in Z_{S_{\infty}}: \mathrm{H}_{S_{\infty}}(z) \leq T\right\}$ and $v_{T}=\mu_{Z_{S_{\infty}}}\left(b_{T}\right)$.

We first claim that there exists $\delta>0$ and $C>0$ such that for any $T>0$,

$$
\int_{b_{T}} \mathrm{H}_{S_{\infty}}^{-k} d \mu_{Z_{S_{\infty}}}<C v_{T}^{1-\delta}
$$

Set $u_{T}$ to be the left hand side of the above inequality. For $T$ large, by Lemma 7.8 (1) one has $v_{T}=O\left(T^{m_{0}}\right)$ for some $m_{0}>0$, hence the derivative $u_{T}^{\prime}=T^{-k} v_{T}^{\prime}$ satisfies $u_{T}^{\prime}=O\left(v_{T}^{-k / m_{0}} v_{T}^{\prime}\right)$ and, integrating, one gets $u_{T}=O\left(v_{T}^{1-k / m_{0}}\right)$. For $T$ bounded, since $\mathrm{H}_{S_{\infty}}$ is bounded below, one gets $u_{T}=O\left(v_{T}\right)$. Putting this together, one gets (13.4).

Now, for any tuple $m=\left(m_{v}\right) \in \mathbb{N}^{S_{f}}$, set $S(m):=\prod S_{v}\left(m_{v}\right) \subset Z_{S_{f}}$ where $S_{v}\left(m_{v}\right):=\left\{z \in Z_{v}:\|z\|_{v}=m_{v}\right\}$.

Also set $\pi_{m}:=\prod_{v} m_{v} \in \mathbb{N}$ and $\omega_{m}=\mu_{Z_{S_{f}}}(S(m))$.

Then we have, where the following sums are taken over the tuples $m \in \mathbb{N}^{S_{f}}$ for which $S(m)$ is non empty,

$$
\begin{aligned}
\int_{B_{T}} \mathrm{H}_{S}(z)^{-k} d \mu_{Z_{S}}(z) & =\sum_{m}\left(\int_{b_{T / \pi}} \mathrm{H}_{S_{\infty}}^{-k} d \mu_{Z_{S \infty}}\right)\left(\int_{S(m)} \mathrm{H}_{S_{f}}^{-k} d \mu_{Z_{S_{f}}}\right) \\
& =\sum_{m} \pi_{m}^{-\frac{k}{2}}\left(\int_{b_{T / \pi}} \mathrm{H}_{S_{\infty}}^{-k} d \mu_{Z_{S_{\infty}}}\right)\left(\int_{S(m)} \mathrm{H}_{S_{f}}^{-\frac{k}{2}} d \mu_{Z_{S_{f}}}\right) \\
& \leq C \sum_{m} \pi_{m}^{-\frac{k}{2}}\left(v_{T / \pi_{m}} \omega_{m}\right)^{1-\delta} \\
& \leq C\left(\sum_{m} \pi_{m}^{-\frac{k}{2 \delta}}\right)^{\delta}\left(\sum_{m} v_{T / \pi_{m}} \omega_{m}\right)^{1-\delta} \\
& \leq C\left(\prod_{v \in S_{f}}\left(1-q_{v}^{-\frac{k}{2 \delta}}\right)\right)^{-\delta} V_{T}^{1-\delta},
\end{aligned}
$$

with positive constants $C$ and $\delta$ given by (13.4) and Corollary 16.7.b.

\section{EFFECTIVE APPLICATIONS}

In this section, assuming $K$ is a number field and $\mathbf{H}$ is a symmetric $K$-subgroup, we give proofs of effective versions of our main theorems listed in the introduction, keeping the notations therein.

Corollary 14.1. Assume that $Z_{\mathcal{O}_{S}} \neq \emptyset$. Then for any finite $v \in S$, there exists $\delta>0$ such that for any effectively well-rounded subset $\Omega \subset Z_{S-\{v\}}$,

$$
\#\left\{z \in Z_{\mathcal{O}_{S}} \cap \Omega:\|z\|_{v}=T\right\}=\widetilde{\operatorname{vol}}\left(\Omega \times S_{v}(T)\right)\left(1+O\left(T^{-\delta}\right)\right)
$$

as $T \rightarrow \infty$ subject to $S_{v}(T) \neq \emptyset$.

Recall that the normalized volume vol has been defined in (1.5). This corollary is an equidistribution statement since one has

$$
\widetilde{\operatorname{vol}}\left(\Omega \times S_{v}(T)\right)=C \mu_{Z_{S-\{v\}}}(\Omega) \mu_{Z_{v}}\left(S_{v}(T)\right)
$$


with a constant $C$ independent of $n$ and $\Omega$.

Proof of Corollary 14.1: The same claim with the error term $O\left(T^{-\delta}\right)$ replaced by $O\left(\left(\mu_{Z_{v}}\left(S_{v}(T)\right)^{-\delta}\right)\right.$ follows immediately from Theorems 11.5, 12.2 and Proposition 13.2. Now by Corollary 16.7. c, we have a constant $a>0$ such that for $T$ large and $S_{v}(T) \neq \emptyset$,

This proves the claim.

$$
\mu_{Z_{v_{0}}}\left(S_{v}(T)\right)^{-1}=O\left(T^{-a / 2}\right)
$$

For $z \in \mathbf{V}_{\mathbb{Q}}$, the condition $\|z\|_{p} \leq p^{n}$ is equivalent to $z \in p^{-n} \mathbf{V}_{\mathbb{Z}}$. Hence we obtain:

Corollary 14.2. Assume $K=\mathbb{Q}$ and fix a prime $p$ such that $Z_{p}$ is non compact and $Z_{\mathbb{Z}\left[p^{-1}\right]}$ is non empty. Then there exists $\delta>0$ such that, for any non-empty compact subset $\Omega \subset Z_{\mathbb{R}}$ with piecewise smooth boundary,

$$
\# \Omega \cap p^{-n} \mathbf{V}_{\mathbb{Z}}=\widetilde{\operatorname{vol}}\left(\Omega \times B_{p}\left(p^{n}\right)\right)\left(1+O\left(p^{-\delta n}\right)\right)
$$

as $n \rightarrow \infty$, where $B_{p}\left(p^{n}\right)$ is defined in (13.1).

Note again that $\widetilde{\operatorname{vol}}\left(\Omega \times B_{p}\left(p^{n}\right)\right)=C \mu_{Z_{\mathbb{R}}}(\Omega) \mu_{Z_{p}}\left(B_{p}\left(p^{n}\right)\right)$ for some $C>0$ independent of $n$ and $\Omega$.

Proof of Theorems 1.3 and 1.7 Letting $v_{T}:=\operatorname{vol}\left(B_{S}(T)\right)$, Theorem 1.3 with $O\left(T^{-\delta}\right)$ replaced by $O\left(v_{T}^{-\delta}\right)$ immediately follows from Theorems 11.5 and 12.2 and Proposition 13.2. To obtain the given error term, note that at least one of the factors $Z_{v_{0}}$ is non-compact. Fix $R_{0}>1$ such that the volume of $B_{S \backslash\left\{v_{0}\right\}}\left(R_{0}\right)=$ $\left\{z \in Z_{S-v_{0}}: \mathrm{H}_{S \backslash\left\{v_{0}\right\}}(z) \leq R_{0}\right\}$ is positive. Since $B_{S}(T)$ contains the product $B_{v_{0}}\left(T R_{0}^{-1}\right) B_{S \backslash\left\{v_{0}\right\}}\left(R_{0}\right)$, we have $v_{T}^{-1}=O\left(\mu_{Z_{v_{0}}}\left(B_{v_{0}}\left(T R_{0}^{-1}\right)\right)^{-1}\right)$. By Corollary 16.3. d for $v_{0}$ archimedean and Corollary 16.7. c for $v_{0}$ non-archimedean, we have a constant $a>0$ satisfying

$$
\mu_{Z_{v_{0}}}\left(B_{v_{0}}(T)\right)^{-1}=O\left(T^{-a / 2}\right) .
$$

This proves the claim. The same proof works for Theorem 1.7 applying Corollary 12.3 in place of Theorem 12.2.

Proof of Corollary 1.10. All three cases fit in our setting as in the introduction.

For $(\mathrm{A})$, if $f$ has signature $(r, s), \mathbf{V}_{1}(\mathbb{R})$ can be identified with $\operatorname{Spin}(r-$ $1, s) \backslash \operatorname{Spin}(r, s)$ where the $\operatorname{Spin}(r, s)$-action on $\mathbb{R}^{r+s}$ is given through the projection $\operatorname{Spin}(r, s) \rightarrow \mathrm{SO}(r, s)$.

For $(\mathrm{B})$ : we have the action of $\mathbf{G}=\mathbf{S L}_{n}$ on $\mathbf{V}$ by $(g, v) \mapsto g^{t} v g$. And $\mathbf{V}_{1}(\mathbb{R})$ is a finite disjoint union of $\mathrm{SO}(r, s) \backslash \mathrm{SL}_{n}(\mathbb{R})$ for $r+s=n$, each of them being the variety consisting of symmetric matrices of signature $(r, s)$.

For $(\mathrm{C})$, we have $\mathbf{V}_{1}(\mathbb{R})=\operatorname{Sp}_{2 n}(\mathbb{R}) \backslash \mathrm{SL}_{2 n}(\mathbb{R})$ with the action $(g, v) \mapsto g^{t} v g$. 
Note for (A), if $n=3, \mathbf{H}_{\mathbb{R}}=\operatorname{Spin}(1,1)$ may arise and the additional assumption that $f$ does not represent 0 over $\mathbb{Q}$ implies that $\mathbf{H}$ does not allow any non-trivial $\mathbb{Q}$-character. In all other cases, $\mathbf{H}$ is semisimple and hence has no character.

Now we give a uniform proof assuming that $S=\{\infty, p\}$ for the sake of simplicity. It is easy to generalize the argument for a general $S$. Also note that this proof works equally well for any homogeneous integral polynomial $f$ whose level set can be identified with a symmetric variety in our set-up.

Let $d=\operatorname{deg} f$. For each $0 \leq j \leq d-1$, consider the radial projection $\pi_{j}$ : $V_{p^{k d+j}} \rightarrow V_{p^{j}}$ given by $x \mapsto p^{-k} x$. Then since the degree of $f$ is $d$ and the radial projection is bijective,

$$
\# \Omega \cap \pi\left(\mathbf{V}_{p^{k d+j}}(\mathbb{Z})^{\operatorname{prim}}\right)=\#\left\{z \in \mathbf{V}_{p^{j}}\left(\mathbb{Z}\left[p^{-1}\right]\right) \cap \Omega_{j}:\|z\|_{p}=p^{k}\right\}
$$

where $\Omega_{j}:=p^{j / d} \Omega \subset \mathbf{V}_{p^{j}}$. Since $\mathbf{V}_{p^{j}}\left(\mathbb{Z}\left[p^{-1}\right]\right)$ is a finite union of $\mathbf{G}\left(\mathbb{Z}\left[p^{-1}\right]\right)$-orbits, we obtain by Corollary 12.3 with $S_{0}=\{\infty\}$ and $S_{1}=\{p\}$

$$
\# \Omega \cap \pi\left(\mathbf{V}_{p^{k d+j}}(\mathbb{Z})^{\text {prim }}\right) \sim \omega_{p^{k d+j}} \operatorname{vol}(\Omega)\left(1+O\left(\omega_{p^{k d+j}}^{-\delta}\right)\right)
$$

where $\omega_{p^{k d+j}}=\mu\left(\left\{x \in \mathbf{V}_{p^{j}}\left(\mathbb{Q}_{p}\right):\|x\|_{p}=p^{k}\right\}\right)$. Note that, by Remark 8.3, $\omega_{m}$ go to infinity with $m$ when it is non zero.

Proof of Corollary 1.11. As before, we assume $S=\{\infty, p\}$ for simplicity. We use the same notation as in the above proof. Then for each fixed $0 \leq j \leq d-1$, $f(x)=p^{k d+j}$ is equivalent to $f\left(p^{-k} x\right)=p^{j}$ and, if $z=p^{-k} x$ with $x \in \mathbf{V}(\mathbb{Z})^{\text {prim }}$, one has $\|z\|_{\infty}\|z\|_{p}=\|x\|_{\infty}$. Therefore

$$
\begin{aligned}
N_{j, T} & :=\#\left\{x \in \mathbf{V}(\mathbb{Z})^{\text {prim }}:\|x\|_{\infty}<T, \quad f(x)=p^{k d+j} \text { for some integer } k \geq 0\right\} \\
& =\#\left\{z \in \mathbf{V}_{p^{j}}\left(\mathbb{Z}\left[p^{-1}\right]\right):\|z\|_{\infty}\|z\|_{p}<T\right\} .
\end{aligned}
$$

By Theorem 1.3, one has $N_{j, T}=v_{j, T}\left(1+O\left(v_{j, T}^{-\delta_{j}}\right)\right)$ where

$$
v_{j, T}=\widetilde{\operatorname{vol}}\left(\left\{\left(z_{\infty}, z_{p}\right) \in \mathbf{V}_{p^{j}}(\mathbb{R}) \times \mathbf{V}_{p^{j}}\left(\mathbb{Q}_{p}\right):\left\|z_{\infty}\right\|_{\infty}\left\|z_{p}\right\|_{p}<T\right\}\right) .
$$

Since $\#\left\{x \in \mathbf{V}(\mathbb{Z})^{\text {prim }}:\|x\|_{\infty}<T, \quad f(x) \in p^{\mathbb{Z}}\right\}=\sum_{j} N_{j, T}$, and $v_{T}=\sum_{j} v_{j, T}$, this proves the claim.

\section{MORE EXAMPLES}

Here are a few concrete examples of applications of Theorem 1.4 to emphasize the meaning of our results. For each of them, we have selected a specific global field $K$ with sets $S_{0}, S_{1}$ (most often $K=\mathbb{Q}, S_{0}=\left\{v_{0}\right\}$ and $S_{1}=\left\{v_{1}\right\}$ ) and we have selected a classical symmetric space $Z$ defined over $K$. We look at the repartition of $S$-integral points $z$ in $Z_{v_{0}}$ when imposing conditions on the $v_{1}$-norm of $z$. 
Symmetric matrices with two real places. This example is very classical. Let $\tau$ be the non trivial automorphism of the real quadratic field $K:=\mathbb{Q}[\sqrt{2}]$ and set, for $d \geq 2$,

$Z_{\{\infty\}}:=\left\{M \in \mathrm{M}_{d}(\mathbb{R})\right.$ positive definite symmetric matrix of determinant 1$\}$ and

$$
Z_{n}:=\left\{M \in Z_{\{\infty\}} \cap \mathrm{M}_{d}(\mathbb{Z}[\sqrt{2}]) \mid \sum_{i, j} \tau\left(m_{i, j}\right)^{2} \leq n^{2}\right\} .
$$

Lemma 15.1. As $n \rightarrow \infty$, these discrete sets $Z_{n}$ become equidistributed in the non-compact Riemannian symmetric space $Z_{\{\infty\}}$.

Proof. Let $v_{0}$ and $v_{1}$ be the two infinite places of $K$ : for $\lambda \in K,|\lambda|_{v_{0}}=|\lambda|$ and $|\lambda|_{v_{1}}=|\tau(\lambda)|$. Apply Theorem 1.4 to

$$
K=\mathbb{Q}[\sqrt{2}], \quad S_{0}=\left\{v_{0}\right\}, \quad S_{1}=\left\{v_{1}\right\}, \quad \mathbf{Z}=\mathbf{S L}_{d} / \mathbf{S O}_{d},
$$

and to the group $\mathbf{G}=\mathbf{S L}_{d}$ which acts by $M \rightarrow g M^{t} g$ on the vector space $\mathbf{V}$ of symmetric $d \times d$-matrices, with $\mathbf{Z} \sim\{M \in \mathbf{V} \mid \operatorname{det}(M)=1\}$ as a G-orbit.

Note that the group $\operatorname{SL}(d, \mathbb{R})$ acts transitively on $Z_{\{\infty\}}$.

Orthogonal projections with one real and one finite place. This example is also quite classical. Let $p$ be a prime number, $d=d_{1}+d_{2} \geq 3$,

$$
Z_{\{\infty\}}:=\left\{\pi \in \mathrm{M}_{d}(\mathbb{R}) \pi^{2}={ }^{t} \pi=\pi \text { and } \operatorname{tr}(\pi)=d_{1}\right\}
$$

the Grassmannian of $\mathbb{R}^{d}$, and

$$
Z_{n}:=\left\{\pi \in Z_{\{\infty\}} \mid p^{n} \pi \in \mathrm{M}_{d}(\mathbb{Z})\right\} .
$$

Lemma 15.2. As $n \rightarrow \infty$, these discrete sets $Z_{n}$ become equidistributed in the compact Riemannian symmetric space $Z_{\{\infty\}}$.

Proof. Apply Theorem 1.4 and Remark 8.3 with

$$
K=\mathbb{Q}, \quad S_{0}=\{\infty\}, \quad S_{1}=\{p\}, \quad \mathbf{Z}=\mathbf{O}_{d} / \mathbf{O}_{d_{1}} \times \mathbf{O}_{d_{2}},
$$

and to the group $\mathbf{G}=\mathbf{S p i n}_{d}$ which acts, by conjugation via $\mathbf{S O}_{d}$, on the vector space $\mathbf{V}$ of $d \times d$-matrices, with $\mathbf{Z} \sim\left\{\pi \in \mathbf{V} \mid \pi^{2}={ }^{t} \pi=\pi\right.$ and $\left.\operatorname{tr}(\pi)=d_{1}\right\}$ as a G-orbit.

Note that the group $\operatorname{Spin}(d, \mathbb{R})$ acts transitively on $Z_{\{\infty\}}$.

Complex structures with one finite and one real place. In this example, one chooses a prime number $p$ and set, for $d \geq 1$,

$$
Z_{\{p\}}:=\left\{J \in \mathrm{M}_{2 d}\left(\mathbb{Q}_{p}\right) \mid J^{2}=-\mathrm{Id} \text { and } \operatorname{tr}(J)=0\right\}
$$

and

$$
Z_{R}:=\left\{J \in Z_{\{p\}} \cap \mathrm{M}_{2 d}\left(\mathbb{Z}\left[\frac{1}{p}\right]\right) \mid \sum_{i, j} J_{i, j}^{2} \leq R^{2}\right\} .
$$

Lemma 15.3. As $R \rightarrow \infty$, these discrete sets $Z_{R}$ become equidistributed in the p-adic symmetric space $Z_{\{p\}}$. 
Proof. Apply Theorem 1.4 to

$$
K=\mathbb{Q}, \quad S_{0}=\{p\}, \quad S_{1}=\{\infty\}, \mathbf{Z}=\mathbf{G L}_{2 d} / \mathbf{G L}_{d} \times \mathbf{G L}_{d},
$$

and to the group $\mathbf{G}=\mathbf{S L}_{2 d}$ which acts by conjugation on the vector space $\mathbf{V}$ of $2 d \times 2 d$-matrices, with $\mathbf{Z} \sim\left\{J \in \mathbf{V} \mid J^{2}=-\mathrm{Id}\right.$ and $\left.\operatorname{tr}(J)=0\right\}$ as a $\mathbf{G}$-orbit.

Note that the group $\mathrm{SL}\left(2 d, \mathbb{Q}_{p}\right)$ acts transitively on $Z_{\{p\}}$.

Antisymmetric matrices with two finite places in characteristic zero. In this example, one chooses two distinct prime numbers $p$ and $\ell$, and set, for $d \geq 1, n \geq 0$, and $R>d$,

$$
Z_{\{p\}}:=\left\{A \in \mathcal{M}_{2 d}\left(\mathbb{Q}_{p}\right) \mid A=-{ }^{t} A \text { and } \operatorname{det}(A)=1\right\}
$$

and

$$
Z_{n, R}:=\left\{A \in Z_{\{p\}} \mid \sum_{i, j} A_{i, j}^{2}<R \text { and } \ell^{n} A \in \mathrm{M}_{2 d}\left(\mathbb{Z}\left[\frac{1}{p}\right]\right)\right\} .
$$

Lemma 15.4. As $n+R \rightarrow \infty$, these discrete sets $Z_{n, R}$ become equidistributed in the p-adic symmetric space $Z_{\{p\}}$.

Proof. Apply Theorem 1.4 and Remark 8.3 with

$$
K=\mathbb{Q}, \quad S_{0}=\{p\}, \quad S_{1}=\{\infty, \ell\}, \quad \mathbf{Z}=\mathbf{S L}_{2 d} / \mathbf{S p}_{d},
$$

and to the group $\mathbf{G}=\mathbf{S L}_{2 d}$ which acts by $g \rightarrow g A^{t} g$ on the vector space $\mathbf{V}$ of antisymmetric $2 d \times 2 d$-matrices, with $\mathbf{Z} \sim\{A \in \mathbf{V} \mid \operatorname{det}(A)=1\}$ as a $\mathbf{G}$-orbit.

Note that the group $\mathrm{SL}_{2 d}\left(\mathbb{Q}_{p}\right)$ acts transitively on $Z_{\{p\}}$.

Quadrics with two places in positive characteristic. In this example, $p$ is an odd prime, and one set, for $d \geq 3$,

$$
Z_{\{0\}}:=\left\{P \in \mathbb{F}_{p}((t))^{d} \mid P_{1}^{2}+\cdots+P_{d}^{2}=1\right\}
$$

and, for $n_{1} \geq n_{2} \geq \ldots \geq n_{d} \geq 0$,

$$
Z_{n_{1}, \ldots, n_{d}}:=\left\{P \in Z_{\{0\}} \cap \mathbb{F}_{p}\left[t, t^{-1}\right]^{d} \mid \operatorname{deg}\left(P_{i}\right)=n_{i} \forall i\right\},
$$

where $\operatorname{deg}\left(\sum a_{i} t^{i}\right):=\max \left\{i \mid a_{i} \neq 0\right\}$.

Lemma 15.5. If $n_{1}=n_{2}=n_{3}$ goes to infinity or if $p \equiv 1 \bmod 4$ and $n_{1}=n_{2}$ goes to infinity, these discrete sets $Z_{n}$ become equidistributed in the sphere $Z_{\{0\}}$.

Proof. Let 0 and $\infty$ be the two (finite) places of the field $\mathbb{F}_{p}(t)$ associated to the two points 0 and $\infty$ of $\mathbb{P}^{1}\left(\mathbb{F}_{p}\right)$. Apply Theorem 1.4 and Remark 8.3 with

$$
K=\mathbb{F}_{p}(t), \quad S_{0}=\{0\}, \quad S_{1}=\{\infty\}, \quad \mathbf{Z}=\mathbf{S O}_{d} / \mathbf{S O}_{d-1},
$$

and to the group $\mathbf{G}=\mathbf{S p i n}_{d}$ which acts naturally, via $\mathbf{S O}_{d}$, on the $d$-dimensional vector space $\mathbf{V}$, with the sphere $\mathbf{Z} \sim\left\{v \in \mathbf{V} \mid v_{1}^{2}+\ldots v_{d}^{2}=1\right\}$ as a $\mathbf{G}$-orbit.

The corresponding two completions are the fields of Laurent series $K_{0}=\mathbb{F}_{p}((t))$ and $K_{\infty}=\mathbb{F}_{p}\left(\left(t^{-1}\right)\right)$, and the ring of $S$-integers is $\mathcal{O}_{S}=\mathbb{F}_{p}\left[t, t^{-1}\right]$. Set

$$
Z_{\{\infty\}}:=\left\{P \in \mathbb{F}_{p}\left(\left(t^{-1}\right)\right)^{d} \mid P_{1}^{2}+\cdots+P_{d}^{2}=1\right\}
$$


note that the well-rounded subset

$$
B_{n_{1}, \ldots, n_{d}}:=\left\{P \in Z_{\{\infty\}} \mid \operatorname{deg}\left(P_{i}\right)=n_{i} \forall i\right\}
$$

is non-empty if and only if $n_{1}=n_{2}=n_{3}$ or $p \equiv 1 \bmod 4$ and $n_{1}=n_{2}$.

Note also that the group $\operatorname{Spin}\left(d, \mathbb{F}_{p}((t))\right)$ acts transitively on $Z_{\{0\}}$.

Other examples. The reader may construct easily many similar examples choosing other triples $(K, S, Z)$. For instance, "Quadrics with three infinite places", "Lagrangian decompositions with two infinite and three finite place", "Hermitian matrices with four places in positive characteristic", and so on....

\section{Appendix: Volume of Balls}

In this appendix we prove precise estimates for the volume of balls which are needed in sections 7 and 13. These estimates will be consequences of the following two general theorems 16.1 and 16.6 .

Volume of balls over the reals. We will first need a variation of a theorem on fiber integration. This theorem says that the volume of the fibers of an analytic function has a, term-by-term differentiable asymptotic expansion in the scale of functions $t^{j}(\log t)^{k}$ with $j$ rational and $k$ non-negative integer. More precisely,

Theorem 16.1. 30] Let $X \subset \mathbb{R}^{m}$ be a smooth real analytic variety, $f: X \rightarrow \mathbb{R}$ a real analytic function and $\nu$ a $C^{\infty}$ measure on $X$. Then, for any compact $K$ of $X$, there exist $d \in \mathbb{N}$ and a set $\left\{A_{j, k}: j \in \frac{1}{d} \mathbb{N}, k \in \mathbb{Z}, 0 \leq k<m\right\}$ of distributions on $X$ supported by $f^{-1}(0)$ such that, for every $C^{\infty}$ function $\varphi: X \rightarrow \mathbb{R}$ with support in $K$, the integral

$$
v_{\varphi}(t):=\int_{0 \leq f(x) \leq t} \varphi(x) d \nu(x)
$$

has a term-by-term differentiable asymptotic expansion when $t>0$ goes to 0

$$
\sum_{j \in \frac{1}{d} \mathbb{N}} \sum_{0 \leq k<m} A_{j, k}(\varphi) t^{j}(\log t)^{k}
$$

This means that, for every $j_{0} \geq 0$, defining $v_{\varphi, j_{0}}$ by truncating the above sum:

$$
v_{\varphi, j_{0}}(t):=\sum_{\substack{0<j \leq j_{0} \\ j \in \frac{1}{d} \mathbb{N}}} \sum_{\substack{0 \leq k<m \\ A_{j, k}}} A_{j} t^{j}(\log t)^{k},
$$

one has $\left(\frac{d}{d t}\right)^{\ell}\left(v_{\varphi}-v_{\varphi, j_{0}}\right)=o\left(t^{j_{0}-\ell}\right)$ for every $\ell \geq 0$.

Remark This theorem is stated for a smooth analytic variety $Z$ and a smooth measure $\mu$. Its proof is based on the real version of Hironaka's resolution of singularity as in [1]. Using once more Hironaka's theorem it can be applied to 
a singular analytic variety $X$ with a measure $\mu$ associated to a meromorphic differential form. Here is one example of such an application:

Proposition 16.2. Let $Z$ be a connected component of the real points of a smooth real affine algebraic variety $\mathbf{Z}$ and $\mu$ a measure on $Z$ which is defined by a regular differential form of $\mathbf{Z}$. Let $F: Z \rightarrow \mathbb{R}$ be a positive proper regular function and set $v_{T}:=\mu(\{z \in Z: F(z) \leq T\})$. Then, there exist positive integers $\ell_{0}$, $d$ and constants $a_{j, k}$ such that $v_{T}$ has a term-by-term differentiable asymptotic expansion when $T \rightarrow \infty$

$$
\sum_{\substack{j \leq \ell_{0} \\ j \in \frac{1}{d} \mathbb{Z}}} \sum_{0 \leq k<m} a_{j, k} T^{j}(\log T)^{k} .
$$

The condition $F$ regular means that $F$ is the restriction to $Z$ of a regular function on the algebraic variety $\mathbf{Z}$.

Proof. Using the resolution of singularities we can view $\mathbf{Z}$ as an open real algebraic subvariety of a smooth projective variety $\mathbf{X}$ such that the boundary $\mathbf{Y}:=\mathbf{X}-\mathbf{Z}$ is a divisor with normal crossing. Hence, in a neighborhood $U_{y_{0}}$ of each real point $y_{0}$ of $\mathbf{Y}$, there is a real local coordinate system $\left(x_{1}, \ldots, x_{m}\right)$ such that $\mathbf{Y}$ is given by $x_{1} \cdots x_{r}=0$, for some $r \leq m$. We are only interested in those points $y_{0}$ in the closure of $Z$. Near these points, the meromorphic function $f:=1 / F$ is zero on $\mathbf{Y}$. Using a partition of unity associated to such a cover, we are reduced to a local problem. Namely proving, for every $C^{\infty}$ function $\varphi$ with compact support in $U_{y_{0}}$, the existence of a term-by-term differentiable asymptotic expansion for

$$
v_{\varphi, \mu}(t):=\int_{f(x) \leq t} \varphi(x) d \mu(x)
$$

when $t:=1 / T$ goes to 0 . It is equivalent to prove the existence of such an asymptotic expansion for the derivative $v_{\varphi, \mu}^{\prime}(t)$ which is called the integral of $\mu$ on the fiber $f^{-1}(t)$.

Using once more the resolution of singularities for the numerator and denominator of $f$ and a new partition of unity, we can assume that $f$ is monomial in these coordinate systems. Using the fact that $f$ is positive on $Z$ and zero on its boundary, we deduce that $f$ is given by $f=s x_{1}^{p_{1}} \cdots x_{r}^{p_{r}}$ with $p_{1}, \ldots, p_{r}$ positive integers and $s= \pm 1$. Hence $f$ is an analytic function near $y_{0}$.

The integral $v_{\varphi, \mu}$ is now very similar to the integral $v_{\varphi}$ of Theorem 16.1 except that $\mu$ may not be smooth. However $\mu$ is defined by a regular differential form on $\mathbf{Z}$ hence there exists a positive integer $\ell_{0}$ such that the measure $\nu:=f^{\ell_{0}} \mu$ is smooth. According to Theorem 16.1, $v_{\varphi, \nu}$ has a term-by-term differentiable asymptotic expansion. The following equality between the fiber integrals

$$
v_{\varphi, \mu}^{\prime}(t)=t^{-\ell_{0}} v_{\varphi, \nu}^{\prime}(t)
$$

implies that $v_{\varphi, \mu}$ has also a term-by-term differentiable asymptotic expansion. 
For us, the main example to which we will apply Proposition 16.2 is a closed orbit $Z$ under the group of $\mathbb{R}$-points of a $\mathbb{R}$-algebraic group, an invariant measure $\mu$ on this orbit and the restriction $F$ to $Z$ of the square of an euclidean norm on $\mathbb{R}^{m}$. Hence we get,

Corollary 16.3. Let $Z$ be a closed orbit of the group $G$ of $\mathbb{R}$-points of an $\mathbb{R}$ algebraic group acting algebraically on a $\mathbb{R}$-vector space $V, \mu$ an invariant measure on $Z$ and $\|\cdot\|$ an euclidean norm on $V$. Set $B_{T}:=\{z \in Z:\|z\| \leq T\}$ and $v_{T}:=\mu\left(B_{T}\right)$.

a) Then $v_{T} \sim c T^{a}(\log T)^{b}$, as $T \rightarrow \infty$, where $a \in \mathbb{Q}_{\geq 0}, b \in \mathbb{Z}_{\geq 0}$ and $c>0$.

b) Moreover $\frac{d}{d T} v_{T} \sim c \frac{d}{d T}\left(T^{a}(\log T)^{b}\right)$, as $T \rightarrow \infty$.

c) For any $k_{0}>0$, there exists $\delta_{0}>0$ such that one has, as $T \rightarrow \infty$,

$$
\int_{B_{T}}\|z\|^{-k_{0}} d \mu(z)=O\left(v_{T}^{1-\delta_{0}}\right) .
$$

d) If $G$ is semisimple and $Z$ is non compact then one has $a \neq 0$.

Remarks - When $Z$ is a symmetric variety, the point a) is proven in [25, Corollary 6.10] for any norm on $V$ and the parameters $a$ and $b$ are explicitly given.

- When $G$ is a group of diagonal matrices, the constant $a$ is zero.

Proof. a) and b) This is a special case of Proposition 16.2. Note that since $v_{T}$ is an increasing function of $T$, one has $a \geq 0$. Moreover, note that, when $a=b=0$, the orbit is of finite volume hence compact.

c) Set $u_{T}:=\int_{B_{T}}\|z\|^{-k_{0}} d \mu(z)$. By a), one has $v_{T}=O\left(T^{m_{0}}\right)$ for some $m_{0}>0$. Hence the derivative $u_{T}^{\prime}=T^{-k_{0}} v_{T}^{\prime}$ satisfies $u_{T}^{\prime}=O\left(v_{T}^{-k_{0} / m_{0}} v_{T}^{\prime}\right)$ and, integrating, one gets $u_{T}=O\left(v_{T}^{1-k_{0} / m_{0}}\right)$.

d) This is a special case of the following Proposition 16.4

Proposition 16.4. With the notations of Corollary 16.3, one has the equivalence: All unipotent elements of $G$ act trivially on $Z \Longleftrightarrow a=0$.

Proof. $\Longrightarrow$ By assumption the normal subgroup of $G$ generated by the unipotent elements of $G$ acts trivially on $Z$. Hence one can assume that $G$ is a product of a compact group by a $r$-dimensional group of diagonal matrices. In this case one has $\mu\left(B_{T}\right)=O\left((\log T)^{r}\right)$ as $T \rightarrow \infty$.

$\Longleftarrow$ This implication is a consequence of the following Lemma 16.5.

Lemma 16.5. Let $U$ be a one-parameter unipotent subgroup of $\mathrm{GL}_{m}(\mathbb{R}), \mu$ a $U$-invariant measure on $\mathbb{R}^{m}$ which is not supported by the $U$-fixed points and set $B_{T}$ for the euclidean ball of radius $T$ on $\mathbb{R}^{m}$. Then one has

$$
\liminf _{T \rightarrow \infty} \frac{\log \left(\mu\left(B_{T}\right)\right)}{\log (T)}>0 .
$$


Proof. First of all, note that all the orbits $U z$ of $U$ in $\mathbb{R}^{m}$ are images of $\mathbb{R}$ by polynomial maps $t \mapsto u_{t} z$ of degree $d_{z} \leq m$. We may assume that this degree $d_{z}$ is $\mu$-almost everywhere non-zero constant. Set $d \geq 1$ for this degree, write $u_{t} z=t^{d} v_{z}+O\left(t^{d-1}\right)$ for some non-zero $v_{z} \in \mathbb{R}^{m}$, and note that the constant involved in this $O\left(t^{d-1}\right)$ is uniform on compact subsets of $\mathbb{R}^{m}$.

One can find a compact subset $C \subset \mathbb{R}^{m}$ transversal to the $U$-action such that $\mu(U C)>0$. The pull-back on $\mathbb{R} \times C$ of the measure $\mu$ by the action $(t, z) \mapsto u_{t} z$ has the form $d t \otimes \nu$ where $d t$ is the Lebesgue measure on $\mathbb{R}$ and $\nu$ is a non-zero measure on $C$. Choose $c>\sup _{z \in C}\left\|v_{z}\right\|$. Then, for $R$ large, one has

$$
u_{[0, R]}(C) \subset B_{c R^{d}}
$$

and hence $\mu\left(B_{c R^{d}}\right) \geq R \nu(C)$. This proves our claim.

Volume of balls over the $p$-adics. We will also need Denef's theorem on $p$-adic integration. For that we need some notations. A subset of $\mathbb{Q}_{p}^{m}$ is said semialgebraic if it is obtained by boolean operations from sets $P_{f, r}:=\{x \in$ $\left.\mathbb{Q}_{p}^{m} / \exists y \in \mathbb{Q}_{p}: f(x)=y^{r}\right\}$ with $f$ a polynomial in $m$ variables with coefficients in $\mathbb{Q}_{p}$ and $r \geq 2$. According to Macintyre's theorem, which is the $p$-adic analog of Tarski-Seidenberg theorem, those sets are exactly the definable sets of the field $\mathbb{Q}_{p}$ [33]. A function $f$ between two $\mathbb{Q}_{p}$-vector spaces is said semialgebraic if its graph is semialgebraic. According to Denef's cell decomposition theorem ([13] and [9]), for every semialgebraic subset $S$, there exists a finite partition of $S$ in semialgebraic sets $S_{1}, \ldots, S_{j_{\max }}$ (called cells) such that, for each $j=$ $1, \ldots, j_{\max }, S_{j}$ is in semialgebraic bijection with a semialgebraic open subset $O_{j}$ of a vector space $\mathbb{Q}_{p}^{d_{j}}$ (recently, R. Cluckers has shown the existence of a semialgebraic bijection between $S$ itself and some $\mathbb{Q}_{p}^{d}$ ). A measure $\mu$ on $S$ is said semialgebraic if there exists a cell decomposition of $S$ on each cell of which $\mu$ is of the form $\left|g_{j}(x)\right| d x$ where $g_{j}$ is a semialgebraic function on $\mathbb{Q}_{p}^{d_{j}}$ and $d x$ is a Haar measure on $\mathbb{Q}_{p}^{d_{j}}$. A function $a: \mathbb{Z} \rightarrow \mathbb{Z}$ is said simple if there are finite partition of $\mathbb{N}$ and $-\mathbb{N}$ by finite sets and arithmetic progressions on which $a$ is affine, see [12, $§ 2.13,2.14$ and 4.4].

Theorem 16.6. [12, Theorem 3.1] Let $\mu$ be a semialgebraic measure on an $m$ dimensional semialgebraic subset $S$ over $\mathbb{Q}_{p}$ and $f$ be a semialgebraic function on S. For $n \in \mathbb{Z}$, set

$$
I_{n}:=\int_{|f(x)|=p^{n}} d \mu(x)
$$

when this integral is finite and $I_{n}=0$ otherwise. Then, for all $n \in \mathbb{Z}$, one has

$$
I_{n}=\sum_{1 \leq i \leq e} \gamma_{i}(n) p^{\beta_{i}(n)}
$$

where $e \in \mathbb{N}, \beta_{i}: \mathbb{Z} \rightarrow \mathbb{Z}$ is a simple function and $\gamma_{i}: \mathbb{Z} \rightarrow \mathbb{Z}$ is a product of at most $m$ simple functions for each $1 \leq i \leq e$. 
For instance, an orbit under the group of $\mathbb{Q}_{p}$-points of a $\mathbb{Q}_{p}$-algebraic group acting algebraically is definable and hence semialgebraic, by Macintyre's theorem, and an invariant measure on this orbit is semialgebraic. Hence one gets:

Corollary 16.7. Let $k$ be a finite extension of $\mathbb{Q}_{p}, q$ the absolute value of an uniformizer, $G$ the group of k-points of an algebraic k-group, $\rho: G \rightarrow G L(V)$ a representation of $G$ defined over $k, Z$ a closed $G$-orbit in $V, \mu$ an invariant measure on $Z$ and $\|\cdot\|$ a max norm on $V$. Denote by $S_{T}$ the sphere $S_{T}=\{z \in$ $Z:\|z\|=T\}$ and set $v_{T}:=\mu\left(S_{T}\right)$.

a) There exists $N_{0} \in \mathbb{N}$ such that, for each $0 \leq j_{0}<N_{0}$, one of the following holds:

(1) $S_{q^{j}}$ is empty, for $j \equiv j_{0} \bmod N_{0}$ large;

(2) there exist $a_{j_{0}} \in \mathbb{Q}_{\geq 0}, b_{j_{0}} \in \mathbb{Z}_{\geq 0}$, and $c_{j_{0}}>0$ such that,

$$
v_{q^{j}} \sim c_{j_{0}} q^{a_{j_{0}} j} j^{b_{j_{0}}} \quad \text { for } j \equiv j_{0} \quad \bmod N_{0} \text { large. }
$$

b) For any $k_{0}>0$, there exists $\delta_{0}>0$ such that one has, as $T \rightarrow \infty$,

$$
\int_{S_{T}}\|z\|^{-k_{0}} d \mu(z)=O\left(v_{T}^{1-\delta_{0}}\right) .
$$

c) If $G$ is semisimple and $Z$ is non compact then, for all $j_{0}$ in case (2), one has $a_{j_{0}} \neq 0$.

Remarks - Let us recall that a max norm is a norm given in some basis $e_{1}, \ldots, e_{m}$ by $\left\|\sum x_{i} e_{i}\right\|=\max \left|x_{i}\right|$.

- When $G$ is a group of diagonal matrices, all the constants $a_{j_{0}}$ are zero.

Proof. Viewing $V$ as a $\mathbb{Q}_{p}$ vector space, we may assume that $k=\mathbb{Q}_{p}$.

a) This is a special case of Theorem 16.6.

b) By a), there exists $m_{0}>0$ such that $v_{T}=O\left(T^{m_{0}}\right)$. Hence one has $\int_{S_{T}}\|z\|^{-k_{0}} d \mu(z)=T^{-k_{0}} v_{T}=O\left(v_{T}^{1-k_{0} / m_{0}}\right)$.

c) This is a special case of the following Proposition 16.8 which is analogous to Proposition 16.4.

Proposition 16.8. With the notations of Corollary 16.7 , the following are equivalent:

(i) All unipotent elements of $G$ act trivially on $Z$,

(ii) For all $j_{0}$ in case (2), one has $a_{j_{0}}=0$,

(iii) Either $Z$ is compact or, for some $j_{0}$ in case (2), one has $a_{j_{0}}=0$.

Proof. The proof is as in Proposition 16.4.

$(i) \Rightarrow($ ii $)$ By assumption the normal subgroup of $G$ generated by the unipotent elements of $G$ acts trivially on $Z$. Hence one can assume that $G$ is a product of a compact group by an $r$-dimensional group of diagonal matrices. In this case, one has $\mu\left(S_{p^{j}}\right)=O\left(j^{r}\right)$ as $j \rightarrow \infty$. 
$($ ii $) \Rightarrow\left(\right.$ iii) If $Z$ is non compact, at least one $j_{0}$ is in case (2).

$($ iii $) \Rightarrow(i)$ This implication is a consequence of the following Lemma 16.9.

Lemma 16.9. Let $k$ be a finite extension of $\mathbb{Q}_{p}, U$ a one-parameter unipotent subgroup of $\mathrm{GL}(m, k), \mu$ a $U$-invariant measure on $k^{m}$ which is not supported by the $U$-fixed points and denote by $S_{T}$ the sphere of radius $T$ on $k^{m}$ for the max norm. Then one has, as $T \rightarrow \infty$ subject to the condition $\mu\left(S_{T}\right) \neq 0$,

$$
\liminf \frac{\log \left(\mu\left(S_{T}\right)\right)}{\log (T)}>0 .
$$

Proof. The proof is as in Lemma 16.5. First of all, note that all the orbits $U z$ of $U$ in $k^{m}$ are images of $k$ by polynomial maps $t \mapsto u_{t} z$ of degree $d_{z} \leq m$. We may assume that this degree $d_{z}$ is $\mu$-almost everywhere non-zero constant. Set $d \geq 1$ for this degree and write $u_{t} z=t^{d} v_{z}+O\left(t^{d-1}\right)$ for some non-zero $v_{z} \in k^{m}$. Let $q$ be the absolute value of an uniformizer. The set $\left\{j \in \mathbb{N}: U z \cap S_{q^{j}} \neq \emptyset\right\}$ is then equal, up to finite sets, to some arithmetic progression $j_{z}+\mathbb{N} d$ with $0 \leq j_{z}<d$. We may assume that this integer $j_{z}$ is $\mu$-almost everywhere constant. Set $j_{0}$ for this integer.

One can find a compact subset $C \subset k^{m}$ transversal to the $U$-action such that $\mu(U C)>0$ and on which $\left\|v_{z}\right\|$ is constant equal to some power $q^{j_{0}+d m_{0}}$ with $m_{0} \in \mathbb{N}$. The pull-back on $k \times C$ of the measure $\mu$ by the action $(t, z) \mapsto u_{t} z$ has the form $d t \otimes \nu$ where $d t$ is a Haar measure on $k$ and $\nu$ is a non-zero measure on $C$. Then, for $|t|=q^{\ell}$ large, one has

$$
u_{t}(C) \subset S_{q^{j_{0}}+d m_{0}+d \ell}
$$

and hence $\mu\left(S_{q^{j_{0}}+d m_{0}+d \ell}\right) \geq(q-1) q^{\ell-1} \nu(C)$. This proves our claim. 


\section{REFERENCES}

[1] M. Atiyah Resolution of singularities and division of distributions, Comm. Pure Appl. Math. 23 (1970) p.145-150.

[2] T. Aubin Nonlinear analysis on manifolds. Monge-Ampère equations, GM 252 Springer (1982).

[3] Y. Benoist Five lectures on lattices Lecture notes (2004).

[4] Y. Benoist, H. Oh Polar decomposition for p-adic symmetric spaces, preprint (2006)

[5] F. Bruhat, J. Tits Groupes réductifs sur un corps local I, Publ. IHES 41 (1972) p.5-252.

[6] F. Bruhat, J. Tits Groupes réductifs sur un corps local II, Publ. IHES 60 (1984) p.5-184.

[7] L. Clozel Démonstration de la conjecture $\tau$, Invent. Math. 151 (2003) 297-328.

[8] L. Clozel, H. Oh and E. Ullmo Hecke operators and equidistribution of Hecke points, Inv. Math. 144 (2003) p.327-351.

[9] R. Cluckers Classification of semialgebraic p-adic sets up to semi-algebraic bijection Jour. Reine Angw. Math. 540 (2001) p.105-114.

[10] S. Dani, G. Margulis Limit distribution of orbits of unipotent flows and values of quadratic forms, Advances in Soviet Math., 16 (1993) p. 91-137

[11] P. Delorme, V. Sécherre An analogue of the Cartan decomposition for $p$-adic reductive symmetric spaces, preprint (2006).

[12] J. Denef On the evaluation of certain p-adic integral Progress in Math. 59 (1985) p25-47

[13] J. Denef $p$-adic semialgebraic sets and cell decomposition Jour. Reine Angw. Math. 369 (1986) p. 154-166.

[14] W. Duke Hyperbolic distribution problems and half integral weight Maass forms Inven. Math. Vol 92 (1988) 73-90.

[15] W. Duke, Z. Rudnick, P. Sarnak Density of integer points on affine homogeneous varieties, Duke Math. Journ. 71 (1993) p. 143-179.

[16] J.Ellenberg, A.Venkatesh Local-Global principles for representations of quadratic forms preprint (2006).

[17] A. Eskin, C. McMullen Mixing, counting and equidistribution in Lie groups, Duke Math. Journ. 71 (1993) p.181-209.

[18] A. Eskin, S. Mozes, N. Shah Unipotent flows and counting lattice points on homogeneous varieties, Annals of Math. 143 (1996) p.149-159.

[19] M. Einsiedler, E.Lindenstrauss ICM, 2006

[20] A.Eskin, H. Oh Representations of integers by an invariant polynomial and unipotent flows, Duke Math. J. 135. (2006) p.481-506.

[21] R. Godement Domaines fondamentaux des groupes arithmétiques Seminaire Bourbaki 257 (1963).

[22] A. Gorodnik, F. Maucourant, H. Oh Manin's and Peyre's conjectures on rational points of bounded height and adelic mixing, preprint (2006).

[23] A. Gorodnik, A. Nevo The ergodic theory of lattice subgroups, preprint (2006)

[24] A. Gorodnik, H. Oh Counting rational points on compactifications of symmetric varieties In preparation.

[25] A. Gorodnik, H. Oh, N. Shah Integral points on symmetric varieties and Satake compactifications, preprint (2006)

[26] A. Gorodnik, B. Weiss Distribution of lattice orbits on homogeneous varieties, To appear in GAFA 
[27] A. Guilloux Equirépartition dans les espaces homogènes, preprint (2006).

[28] A. Helminck, S. Wang On rationality properties of involutions of reductive groups, Adv. in Math. 99 (1993) p.26-97.

[29] H. Iwaniec Fourier coefficients of modular forms of half integral weight, Inv. Math (1987), Vol 87, pp. 385-401.

[30] P. Jeanquartier Integration sur les fibres d'une fonction analytique Travaux en Cours 34 (1988) p.1-39.

[31] F. Ledrappier Distribution des orbites des réseaux sur le plan réel, CRAS 329 (1999) p.6164.

[32] Y. V. Linnik Additive problems and eigenvalues of the modular operators, Proc. Int. Cong. Math. Stockholm, 1962, pp. 270-284.

[33] A. Macintyre, On definable subsets of p-adic fields, J. Symb. Logic 41 (1976) p.605-610.

[34] G. Margulis, On some aspects of the theory of Anosov systems, Springer (2004).

[35] G. Margulis Discrete subgroups of semisimple Lie groups, Springer Ergebnisse (1991).

[36] P. Michel, A. Venkatesh ICM 2006

[37] F. Maucourant Homogeneous asymptotic limits of Haar measures of semisimple linear groups, To appear in Duke M. J.

[38] S. Mozes, N. Shah On the space of ergodic invariant measures of unipotent flows, ETDS 15 (1995) p. 149-159

[39] H. Oh Uniform pointwise bounds for matrix coefficients of unitary representations and applications to Kazhdan constants, Duke Math. J. 113 (2002) p.133-192.

[40] H. Oh Hardy-Littlewood system and representations of integers by invariant polynomials, GAFA, 14 (2004) p. 791-809

[41] G. Prasad Strong approximation for semisimple groups over function fields, Annals of Math., 105 (1977) 553-572

[42] V. Platonov, A. Rapinchuk Algebraic groups and number theory, Ac. Press (1994).

[43] M. Ratner On Raghunathan's measure conjecture, Ann. of Math., Vol 134, 1991, pp. 545607.

[44] P. Sarnak Diophantine problems and linear groups, Proc. Int. Cong. Math. 1990, Vol 1, pp. 459-471.

[45] N. Shah. Limit distribution of expanding translates of certain orbits on homogeneous spaces on homogeneous spaces, Proc. Indian Acad. Sci. Math. Sci. 106 (1996), no. 2, 105-125.

DMA-ENS 45 RUE D'UlM PARIS 75005

E-mail address: benoist@dma.ens.fr

Mathematics 253-37, Caltech, Pasadena, CA 91125

current address: Mathematics, Box 1914, Brown University, Providence, RI

E-mail address: heeoh@math.brown.edu 